\title{
Basal Contamination of Sequencing: Lessons from the GTEx dataset
}

Tim O. Nieuwenhuis ${ }^{1,2}$, Stephanie Yang ${ }^{2}$, Rohan X. Verma ${ }^{1}$, Vamsee Pillalamarri $^{2}$, Dan E. Arking ${ }^{2}$, Avi Z. Rosenberg ${ }^{1}$, Matthew N. McCall ${ }^{3}$, Marc K. Halushka ${ }^{1 *}$

${ }^{1}$ Department of Pathology, Johns Hopkins University SOM, Baltimore, MD, USA

${ }^{2}$ McKusick-Nathans Institute, Department of Genetic Medicine, Johns Hopkins University SOM, Baltimore, MD, USA

${ }^{3}$ Department of Biostatistics and Computational Biology, University of Rochester Medical Center, Rochester, NY, USA

\section{Email Addresses:}

tnieuwe1@jhmi.edu

syang93@jhmi.edu

rverma6@jhmi.edu

vpillal1@jhmi.edu

arking@jhmi.edu

arosen34@jhmi.edu

matthew_mccall@urmc.rochester.edu

* Correspondence and address for reprints to: Marc K. Halushka, M.D., Ph.D.

Johns Hopkins University School of Medicine

Ross Bldg. Rm 632B

720 Rutland Avenue

Baltimore, MD 21205

410-614-8138 (ph)

410-502-5862 (fax)

mhalush1@jhmi.edu 


\section{Abstract}

One of the challenges of next generation sequencing (NGS) is read contamination. We used the Genotype-Tissue Expression (GTEx) project, a large, diverse, and robustly generated dataset, to understand the factors that contribute to contamination. We obtained GTEx datasets and technical metadata and validating RNA-Seq from other studies. Of 48 analyzed tissues in GTEx, 26 had variant co-expression clusters of four known highly expressed and pancreas-enriched genes (PRSS1, PNLIP, CLPS, and/or CELA3A). Fourteen additional highly expressed genes from other tissues also indicated contamination. Sample contamination by non-native genes was associated with a sample being sequenced on the same day as a tissue that natively expressed those genes. This was highly significant for pancreas and esophagus genes (linear model, $p=9.5 e-237$ and $p=5 e-260$ respectively). Nine SNPs in four genes shown to contaminate non-native tissues demonstrated allelic differences between DNA-based genotypes and contaminated sample RNA-based genotypes, validating the contamination. Low-level contamination affected 4,497 (39.6\%) samples (defined as $\geq 10$ PRSS1 TPM). It also led to eQTL assignments in inappropriate tissues among these 18 genes. We note this type

51 of contamination occurs widely, impacting bulk and single cell data set analysis. In

52 conclusion, highly expressed, tissue-enriched genes basally contaminate GTEx and

53 other datasets impacting analyses. Awareness of this process is necessary to avoid

54 assigning inaccurate importance to low-level gene expression in inappropriate tissues 55 and cells.

56 Key Words: GTEx, RNA-Seq, Contamination, scRNA-Seq, eQTL, PEER factors 


\section{Introduction}

The rise of next generation sequencing has allowed for unparalleled data generation for a variety of nucleic acid studies including RNA expression. As cost per basepair decreases, more large-scale transcriptome projects can be performed that will inform on tissue expression patterns in health and disease ${ }^{1-4}$. These data sources are generally publicly-available and have been used by hundreds of researchers for secondary analyses of high impact ${ }^{5,6}$.

Limitations exist for all -omics technologies, including RNA sequencing (RNASeq). Issues of hybridization biases, library preparation biases, and computational biases such as positional fragment bias are known limitations of RNA-Seq experiments ${ }^{7-}$ ${ }^{9}$. Another challenge of high throughput RNA-Seq is contamination, leading to the presence of sequence data within a dataset of one sample that originates from a separate sample. This contamination can come from many different aspects of the modern sequencing process, such as human error, machine or equipment contamination, intrinsic preparation and sequencing errors, and computational errors, including errors that can occur based on the multiplexing methods used ${ }^{10-12}$. For single cell RNA-Seq (scRNA-Seq), doublets or multiplets (2+ cells partitioned together) can cause cross-contamination resulting in expression hybrids ${ }^{13}$. Compared to RNA-Seq, contamination has been better characterized for DNA sequencing projects ${ }^{14-16}$.

The Genotype-Tissue Expression project (GTEx) aims to create a large publicly available database of tissue-specific expression quantitative trait loci (eQTL) from over 40 tissues $^{1}$. It is an ongoing project with over 700 individuals and 11,000 tissue samples. GTEx identifies eQTLs by associating genotypes called from whole genome 
81

82

sequencing with gene expression levels obtained from bulk RNA-Seq. GTEx has made their RNA-Seq, phenotype, genotype, and technical data available for public access with permission.

In an analysis of variation in the GTEx RNA-Seq data (V7), we detected unexpected sources of variation that we hypothesized were likely contaminating sequence reads found at low, but variable levels across different tissues. Herein we describe how we identified the source of contamination and establish basal rates of contamination in the GTEx bulk RNA-Seq data. We further demonstrate the universality of highly-expressed genes contaminating other samples.

\section{Results}

\section{Patterns of extreme tissue variation identified usual and unusual gene signatures}

We embarked on a project to expand our initial description of the causes of lung expression variation in GTEx to all tissue samples ${ }^{17}$. We used DESeq2 variance stabilizing transformation to normalize read counts from 11,340 samples $^{17,18}$. Then we filtered genes in each tissue keeping those with a mean transformed count $>5$. The median number of genes above the expression threshold was 17,729 with the highest and lowest gene counts being 23,930 and 13,807 in the testis and whole blood respectively. As previously described, we correlated and hierarchically clustered variable genes ( $>4$ variance across samples) for all tissues with $>70$ samples $(N=48)$ in the GTEx dataset $V 7{ }^{17}$. Our algorithm identified multiple gene clusters per tissue, based on their Kendall's tau correlations (Fig. 1a). It additionally reported nonclustering, highly variable genes. Most clusters were the result of biologic and 
102 phenotypic features related to the tissues. For example, a cluster of $\mathrm{Y}$ chromosome

103 genes and XIST appeared in 42 of 43 non-sex specific tissues. However, there was one

104 consistent cluster of 3-4 genes (PNLIP, PRSS1, CELA3A, and/or CLPS) identified in 26

105 of the 48 tissues that did not have an intuitive biological explanation. These genes are

106 highly-expressed and pancreas acinar-cell specific ${ }^{19}$. To identify other highly expressed

107 tissue-enriched genes appearing variably in other samples, we cross-referenced a list of

108 tissue-enriched proteins generated by the Human Protein Atlas (HPA) to the GTEx

109 transcripts per million (TPM) data (Table 1$)^{20,21}$. We noted 18 genes from seven tissues

110 including two esophagus genes KRT13 and KRT4 that are highly expressed in their

111 native tissue and identified as variable in five or more other unrelated tissues (Fig. 1a,

112 Supplementary Fig. 1).

113 Table 1 Eighteen highly expressed genes that appear to contaminate five or more

114 GTEx tissues

\begin{tabular}{|l|c|l|c|c|c|c|}
\hline \multirow{2}{*}{ Gene } & \multirow{2}{*}{$\begin{array}{c}\text { Times } \\
\text { identified as } \\
\text { variable in }\end{array}$} & $\begin{array}{c}\text { Highest } \\
\text { expressed } \\
\text { other tissues }\end{array}$ & GTEx tissue & \multirow{2}{*}{$\begin{array}{c}\text { GTEx } \\
\text { TPM }\end{array}$} & \multicolumn{2}{|c|}{ 2nd Highest expressed GTEx Tissue } \\
\cline { 5 - 7 } & 43 & Pancreas & 99,100 & Tissue & GTEx TPM & $\begin{array}{c}\text { Independent } \\
\text { Study TPM }\end{array}$ \\
\hline PRSS1 & 34 & Pancreas & 33,660 & Ovary & 3.67 & 0.92 \\
\hline PNLIP & 31 & Pancreas & 54,500 & Testis & 8.16 & 0 \\
\hline GPA1 & 29 & Pancreas & 14,280 & Prostate & 18.34 & 13.5 \\
\hline CELA3A & 24 & Pancreas & 27,130 & Stomach & 14.1 & 0.03 \\
\hline KRT13 & 20 & Esophagus & 33,960 & Vagina & 13,140 & 3,961 \\
\hline PGC & 19 & Stomach & 36,720 & Lung & 83.84 & 12.42 \\
\hline KRT4 & 18 & Esophagus & 22,290 & Vagina & 1,375 & 15,069 \\
\hline PRL & 17 & Pituitary & 54,500 & Testis & 5.15 & 0 \\
\hline LIPF & 15 & Stomach & 29,380 & Testis & 4.33 & 0 \\
\hline CLPS & 14 & Pancreas & 51,640 & Stomach & 5.35 & 0 \\
\hline CTRB2 & 9 & Pancreas & 20,760 & Ovary & 3.21 & 0 \\
\hline MYBPC1 & 6 & $\begin{array}{l}\text { Skeletal } \\
\text { muscle }\end{array}$ & 3,587 & Prostate & 54.5 & 21.3 \\
\hline
\end{tabular}


bioRxiv preprint doi: https://doi.org/10.1101/602367; this version posted January 2, 2020. The copyright holder for this preprint (which was not certified by peer review) is the author/funder, who has granted bioRxiv a license to display the preprint in perpetuity. It is made available under aCC-BY-NC-ND 4.0 International license.

\begin{tabular}{|l|c|l|c|c|c|c|}
\hline MYH2 & 6 & $\begin{array}{l}\text { Skeletal } \\
\text { muscle }\end{array}$ & 1,064 & Colon & 0.52 & 0.02 \\
\hline ZG16B & 6 & $\begin{array}{l}\text { Salivary } \\
\text { gland }\end{array}$ & 17,540 & Prostate & 48.1 & 187.1 \\
\hline FGA & 5 & Liver & 5,717 & Stomach & 12.43 & 6.02 \\
\hline$H P$ & 5 & Liver & 12,710 & Adipose & 140.1 & 2.7 \\
\hline CKM & 5 & $\begin{array}{l}\text { Skeletal } \\
\text { muscle }\end{array}$ & 11,138 & Heart & 2,987 & 2,339 \\
\hline $\begin{array}{l}\text { Independent study samples were taken from RNA-seq experiments of the } 2^{\text {nd }} \text { highest GTEx } \\
\text { tissue and were not co-sequenced with the highest expressed GTEx tissue. }\end{array}$ \\
\hline \multicolumn{7}{l}{} \\
\hline
\end{tabular}

a

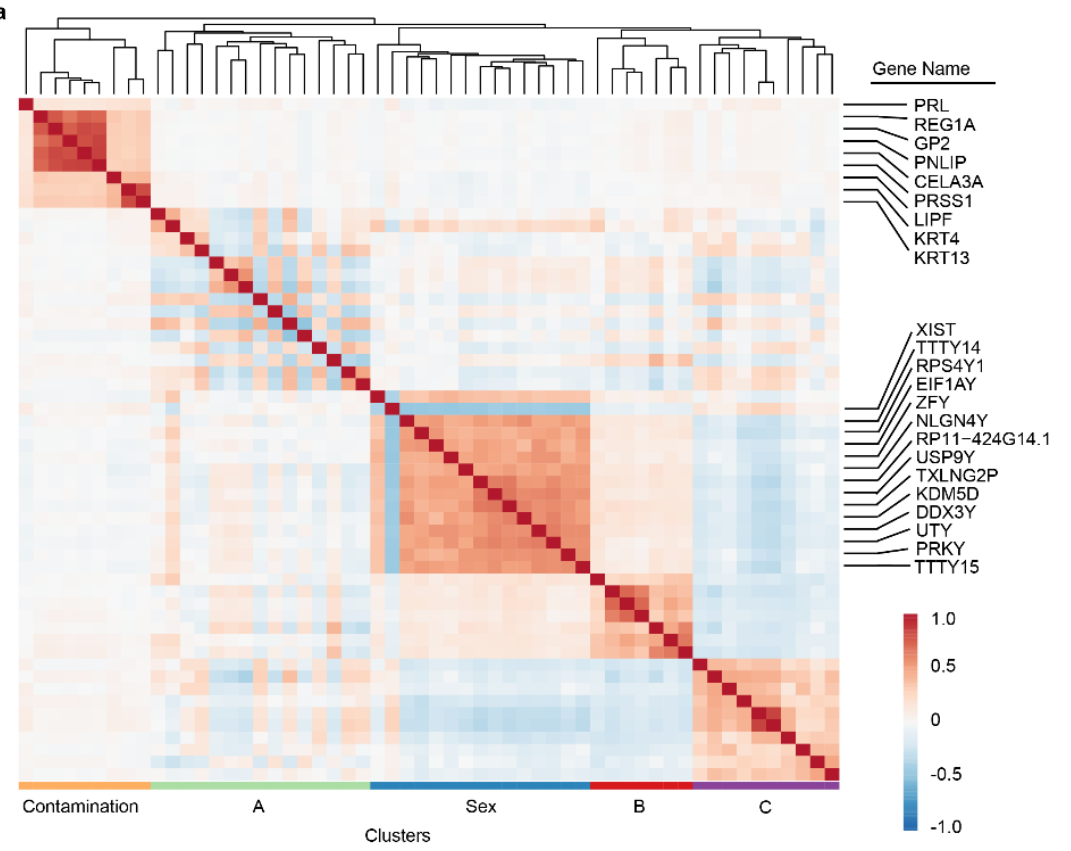

d

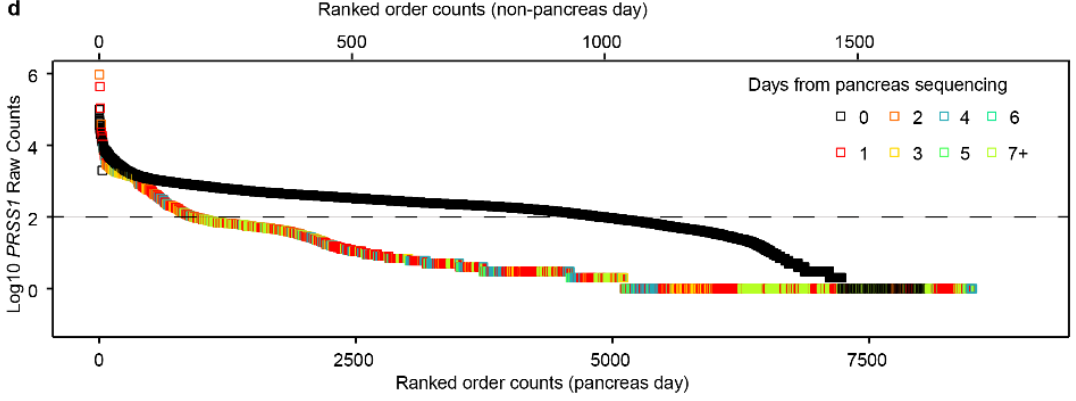

b
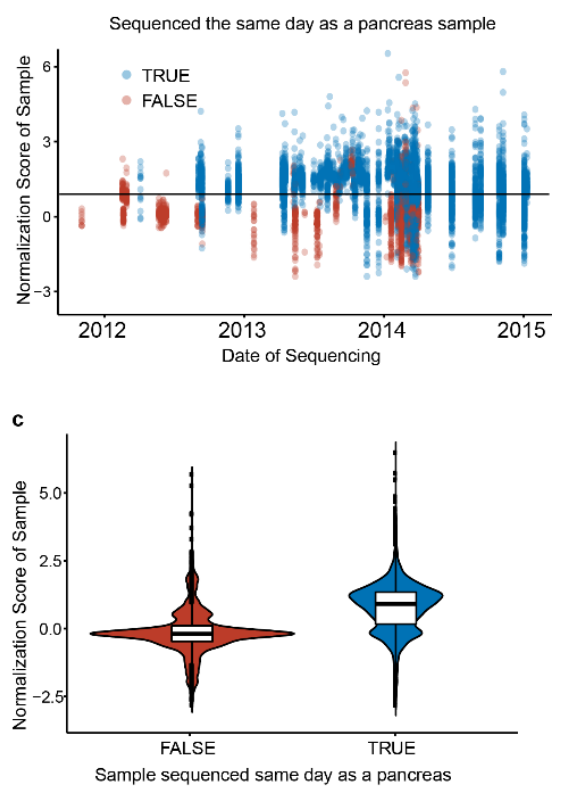

e

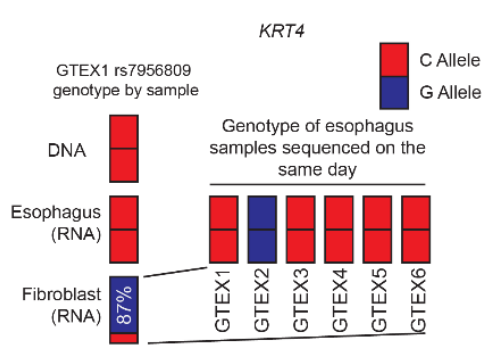


117 Fig. 1 Identification and explanation of sequencing contamination a A correlation heatmap of highly variable subcutaneous adipose tissue genes across 442 subjects. Red shows a positive correlation. The genes within the contamination cluster and the sex cluster are given. The meaning of cluster $A$ is unknown. Cluster B may relate to the

121 percentage of smooth muscle cells and cluster $\mathbf{C}$ includes acute phase reactants $\mathbf{b}$

122 Contamination normalized score values for non-pancreas tissue samples $(\mathrm{N}=11,366)$

123 colored relative to being sequenced on the same day as a pancreas tissue. c Violin plot

124 of the same data showing a strong, but not complete correlation of sequencing on a

125 pancreas day. d Ranked order of all samples either sequenced on the same day as a

126 pancreas sample (black) or on a non-pancreas sequencing day (colors) for PRSS1 read

127 counts in log10. Among samples not sequenced on a pancreas day, 91\% of samples

128 with $>100$ reads were sequenced within 4 days of a known sequenced pancreas. The

129 dashed line represents 100 reads. e KRT4 contaminating reads in GTEX1's fibroblast

130 RNA-Seq appear to have originated from GTEX2 esophagus mucosa tissue. By DNA

131 and RNA of the appropriate tissue source of KRT4, sample GTEX1 is homozygous for

132 the $\mathrm{C}$ allele at $\mathrm{rs} 7956809$. The fibroblast sample is $87 \% \mathrm{G}$ reads, primarily matching

133 sample GTEX2. The read count depth at the SNP in the GTEX1 esophagus was 85,803

134 and 204 for the GTEX1 fibroblast.

As both abundant and tissue-enriched genes were unlikely to be randomly and

136 lowly expressed in a range of other tissues, we performed analyses to determine the

137 source of the contamination.

We first questioned if the contamination occurred during tissue harvesting,

139 hypothesizing that occasionally small fragments of a tissue could contaminate a 
separate sample from shared dissection tools or surfaces. A pancreas gene contamination cluster was found in transformed fibroblasts which were grown over multiple passages and would not retain other cell types over that time period, challenging this as a source of contamination (Supplementary Fig. 1).

\section{Linear mixed models reveal sequencing date as the source of contamination}

We then queried if other technical sources of contamination may be related. Other GTEx metadata available included nucleic acid isolation date and sequencing date, which were both interrogated for associations with this contamination. The normalized score of four pancreas-enriched genes (PRSS1, CLPS, PNLIP, and CELA3A) was significantly higher in non-pancreas samples if they were sequenced on the same day as a pancreas sample (Wilcox Rank Sum Test, $p<5 e-324$, Fig 1b,c). We then performed linear mixed model analyses across non-contaminating tissues to understand expression levels of genes known to be highly-enriched and highlyexpressed in the pancreas or esophagus, but were found lowly and variably in other tissues (ex. coronary artery, liver, tibial nerve). Because the majority of samples were sequenced on the same day as a pancreas or esophagus - mucosa sample, for model robustness, we limited our models to only tissues with $>40$ samples not sequenced on the same data as a respective contaminating tissue (Supplementary Data 1 and 2).

After adjusting for tissue type, the pancreas contamination score (average of normalized expression values for PRSS1, CLPS, PNLIP, and CELA3A) was on average 0.175 higher if the tissue was isolated on the same day a pancreas sample $(p=3.9 e-12)$, but on average 0.863 higher if the tissue was sequenced on the same day as a pancreas sample ( $p=9.5 e-237)$ (Supplementary Data 3). 
A similar linear mixed model was used to evaluate the esophagus contamination

164

165

166

167 179 (Supplementary Figure 2).

(Supplementary Data 4).

score (average of normalized expression values for two highly-expressed esophagus-

enriched genes KRT4 and KRT13). The same model as above showed only sequencing

a tissue on the same day as a esophagus sample to be significant $(p=5 e-260)$

Despite the significance of with sequencing date, some high scores came from samples that were not sequenced on the same days as pancreata. Focusing on just one these genes, PRSS1, it was clear that all of these samples were sequenced within a few days of a pancreas (Fig. 1d). This additionally implicated the library preparation process (for which date information is lacking in GTEx) as a potential source for contamination, as it is temporally related, but not identical, to sequencing date.

With this understanding of the temporal importance of sequencing date to gene expression, we then revisited the four genes making up the pancreas normalized score gene cluster. For all tissues, median normalized scores were higher on pancreas sequencing dates, and the division between the 26 tissues with clustering vs. the 21 without was a result of the high threshold for TPM we set at the VST step of our pipeline

\section{Genetic polymorphisms confirm contamination is derived from other samples}

To prove that pancreas/esophagus transcripts were contaminating from other

182 (non-self) samples we identified incongruencies between a person's genotype (from 
185 from contaminating tissues, in order to account for both RNA editing and preferential

186 allele expression. Based on these sample requirements and limited by available raw

187 sequencing files, we identified 11 contaminated tissues to evaluate. For each, we

188 obtained and processed their raw RNA-Seq FASTQ sequences to identify nucleotide

189 variants in both their contaminated tissues and their matched pancreas or esophagus

190 tissue (depending on the gene source of contamination). Additionally, we used the

191 GTEx filtered VCF file from the individual's sequenced DNA to further establish their

192 SNP allele patterns. Across all tissues, 533 SNPs, rare variants, and private variants,

193 were investigated in pancreas associated gene coding sequences (PNLIP, CLPS, and

194 CELA3A) and 190 in esophagus associated gene coding sequences (KRT13, KRT4).

195 As a comparison group, 287 variants were investigated in two control gene coding

196 sequences (GAPDH, and $R A B 7 A)$ that have near ubiquitous expression across all

197 tissues. Of 1,010 variants obtained from the combined VCF files, 11 had some degree

198 of allelic heterogeneity (Table 2). No incongruencies were found in the 287 variants of

199 the two control genes.

Table 2 Allelic inconsistencies found in contaminated samples

\begin{tabular}{|c|c|c|c|c|c|c|c|c|}
\hline \multirow[b]{2}{*}{ Individual } & \multirow[b]{2}{*}{ Gene } & \multirow[b]{2}{*}{ SNP } & \multicolumn{3}{|c|}{ Enriched Tissue } & \multicolumn{3}{|c|}{ Contaminated } \\
\hline & & & $\begin{array}{c}\text { Major/ } \\
\text { Minor }\end{array}$ & Reads & $\begin{array}{c}\text { Major } \\
\text { Allele \% }\end{array}$ & Tissue Type & Reads & $\begin{array}{l}\text { Major } \\
\text { Allele \% }\end{array}$ \\
\hline GTEX-1 & KRT13 & rs903 & $\mathrm{C} / \mathrm{A}$ & 101,908 & $0 \%$ & Fibroblast Cells & 252 & $50 \%$ \\
\hline GTEX-1 & KRT4 & rs7959052 & $\mathrm{T} / \mathrm{C}$ & 74,468 & $100 \%$ & Fibroblast Cells & 203 & $12 \%$ \\
\hline GTEX-1 & KRT4 & rs7956809 & $C / G$ & 85,803 & $100 \%$ & Fibroblast Cells & 204 & $13 \%$ \\
\hline GTEX-1 & KRT4 & rs2035879 & $\mathrm{T} / \mathrm{C}$ & 72,978 & $51 \%$ & Fibroblast Cells & 164 & $7 \%$ \\
\hline GTEX-1 & KRT4 & rs17119475 & $\mathrm{G} / \mathrm{A}$ & 71,592 & $49 \%$ & Fibroblast Cells & 226 & $98 \%$ \\
\hline GTEX-8 & CELA3A & rs9187 & $\mathrm{C} / \mathrm{T}$ & 162,318 & $73 \%$ & Tibial Nerve & 1,155 & $100 \%$ \\
\hline GTEX-8 & CELA3A & rs12908 & $\mathrm{G} / \mathrm{A}$ & 169,394 & $74 \%$ & Tibial Nerve & 1,215 & $100 \%$ \\
\hline GTEX-9 & CELA3A & rs3820285 & $\mathrm{C} / \mathrm{G}$ & 98,896 & $1 \%$ & Adipose & 5,178 & $48 \%$ \\
\hline GTEX-9 & $C E L A 3 A$ & rs9187 & $\mathrm{C} / \mathrm{T}$ & 105,462 & $75 \%$ & Adipose & 6,082 & $97 \%$ \\
\hline GTEX-9 & $C E L A 3 A$ & rs12908 & $\mathrm{G} / \mathrm{A}$ & 108,681 & $75 \%$ & Adipose & 6,313 & $98 \%$ \\
\hline
\end{tabular}




\begin{tabular}{l|l|l|l|l|l} 
GTEX-10 & CLPS & rs3748050 & T/C & 80,019 & $47 \% \mathrm{C}$
\end{tabular}

One SNP site, rs7956809, was particularly informative. SNP rs7956809 (C/G),

203 located in KRT4, had a relatively low allelic variation, with only 5 individuals in the entire

204 GTEx cohort homozygous for the alternative allele (G). One individual (arbitrarily

205 GTEX1) was homozygous C at rs7956809 in both its DNA (VCF file) and matched

206 esophagus (RNA-Seq FASTQ data) (Fig. 1e). However, the rs7956809 SNP in the

207 GTEX1 fibroblast sample was $87 \%$ G and $13 \%$ C. Six esophagus samples were

208 sequenced on the same day as the GTEX1 fibroblast sample. No other esophagus

209 samples were sequenced within 4 days. One of those six samples, GTEX2, was

210 homozygous $\mathrm{G}$ at rs7956809. The five other samples were homozygous $\mathrm{C}$. This

211 strongly implicates the GTEX2 esophagus sample as the dominant contaminant of the

212 GTEX1 fibroblast sample.

We further investigated the relationship between the GTEX1 fibroblast sample

214 and the GTEX2 esophagus sample finding no clear connection. The two samples were

215 sequenced on different machines and in different flow cells. Of some interest, the

216 sequencing sample adapters (molecular indexes) were similar (Supplementary Data 5).

217 The extent of highly expressed, tissue-enriched gene contamination in GTEx 
218 After establishing that contamination exists in GTEx by identification of a temporal

219 association and validation through polymorphisms, we then attempted to address the

220 extent of contamination in the GTEx dataset. To characterize this, we investigated the

221 various levels of pancreas enhanced gene expression in non-pancreatic tissue (Fig. 2).

222 In the 11,366 non-pancreas samples investigated, roughly $25 \%$ had 0 reads for each of

2234 pancreas-enriched genes and another $\sim 50 \%$ of samples had TPM $<10$.

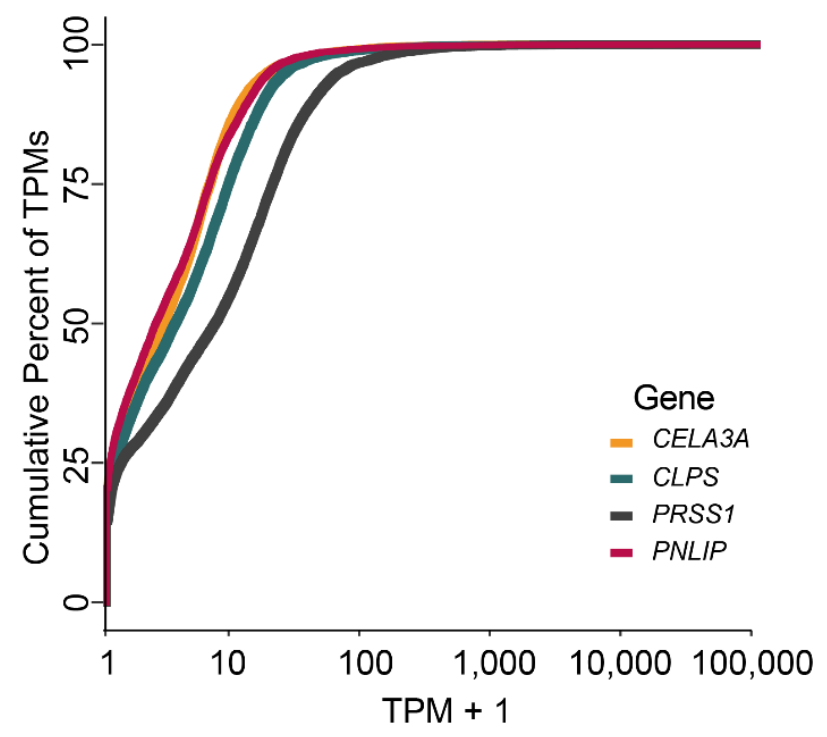

225 Fig. 2 A density plot of 11,366 non-pancreas RNA-seq samples and their cumulative

226 TPM expression of four pancreas genes.

228 The GTEx analysis pipeline uses probabilistic estimation of expression residuals

229 (PEER) factor to correct for possible confounders ${ }^{22,23}$. This method identifies hidden

230 factors that explain much of the expression variability and can be used to normalize

231 RNA expression data. We focused on just one tissue, lung, and followed the GTEx

232 analysis pipeline to determine the extent to which PEER factor normalization can 
233 identify and correct for this contamination. Sixty PEER factors were identified, with the

234 top two capturing a difference between "in hospital" (short postmortem interval) and

235 "outside of hospital" (longer postmortem interval) deaths (Fig. 3a). This relationship is

236 consistent with our prior report of variation in lung $^{17}$. Similar to the global findings of Fig.

237 1, PNLIP expression was increased in lung samples sequenced on the same day as a

238 pancreas. Despite correcting for 35 or even 60 PEER factors, this difference was not

239 fully accounted for (Fig. 3b). Indeed, of five genes evaluated, only one gene (KRT4)

240 was fully corrected for by PEER factors (Table 3). We then explored if this lack of full

241 correction impacted eQTL analysis in the GTEx program.

242 Table 3. Significance of same-day sequencing of lung with contaminating tissues

243 on gene expression

\begin{tabular}{|l|r|r|r|r|}
\hline \multicolumn{1}{|c|}{ Gene } & $\begin{array}{c}\text { P. value } \\
\text { before PEER } \\
\text { correction }\end{array}$ & $\begin{array}{c}\text { P. value after } \\
\text { correcting for } \\
\text { 35 PEER } \\
\text { factors }\end{array}$ & $\begin{array}{c}\text { P. value after } \\
\text { correcting for } \\
\text { 60 PEER } \\
\text { factors }\end{array}$ & $\begin{array}{c}\text { Beta estimate } \\
\text { after } \\
\text { correction }\end{array}$ \\
\hline PNLIP & $4.34 \mathrm{e}-14$ & $1.39 \mathrm{e}-07$ & $1.94 \mathrm{e}-05$ & 0.49 \\
\hline PRSS1 & $6.29 \mathrm{e}-14$ & $5.83 \mathrm{e}-07$ & $2.63 \mathrm{e}-05$ & 0.48 \\
\hline CELA3A & $5.91 \mathrm{e}-14$ & $3.36 \mathrm{e}-07$ & $2.41 \mathrm{e}-05$ & 0.49 \\
\hline KRT4 & 0.0034 & 0.17 & 0.15 & 0.18 \\
\hline KRT13 & $8.18 \mathrm{e}-17$ & 0.00048 & 0.0036 & 0.37 \\
\hline P. & & & \\
\hline
\end{tabular}

P. values are shown before and after PEER correction. 
a
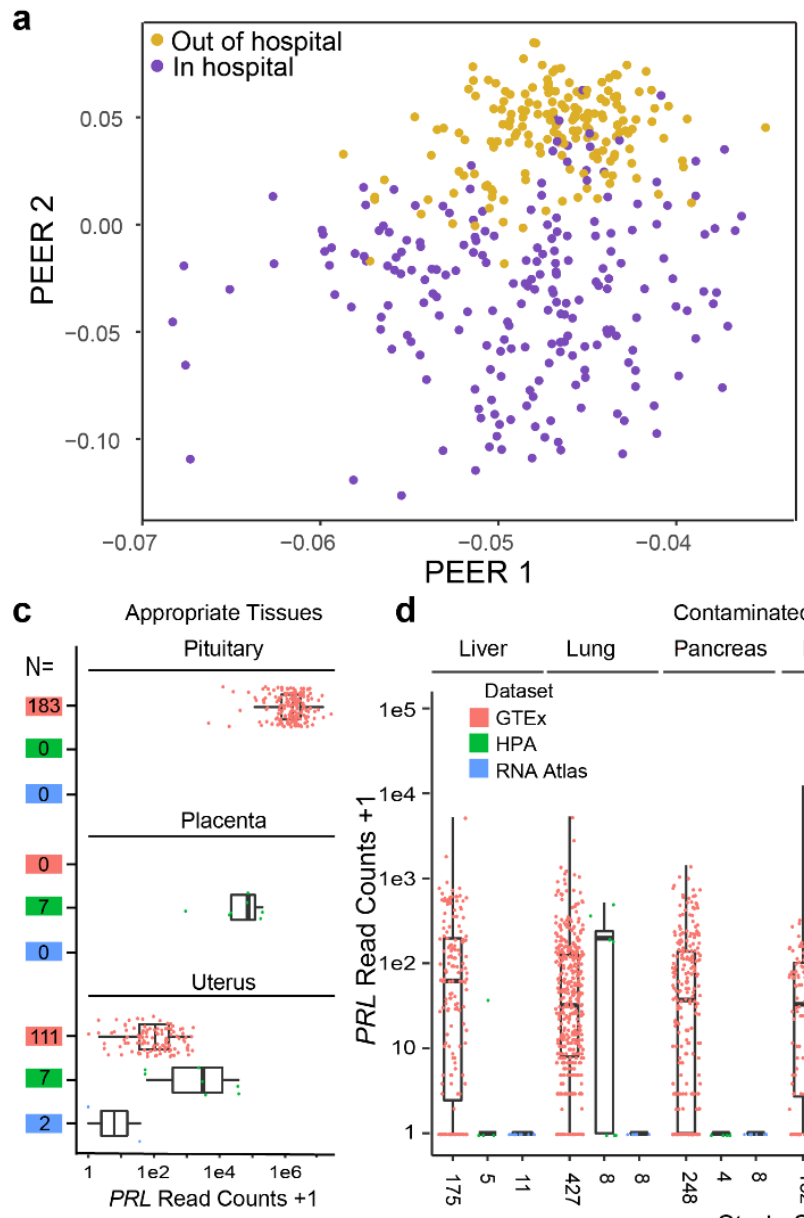

244

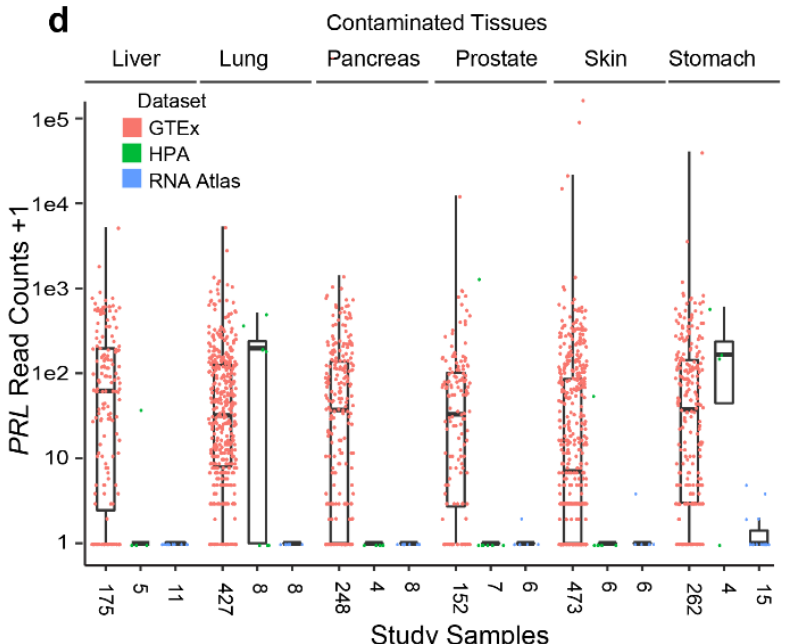

b

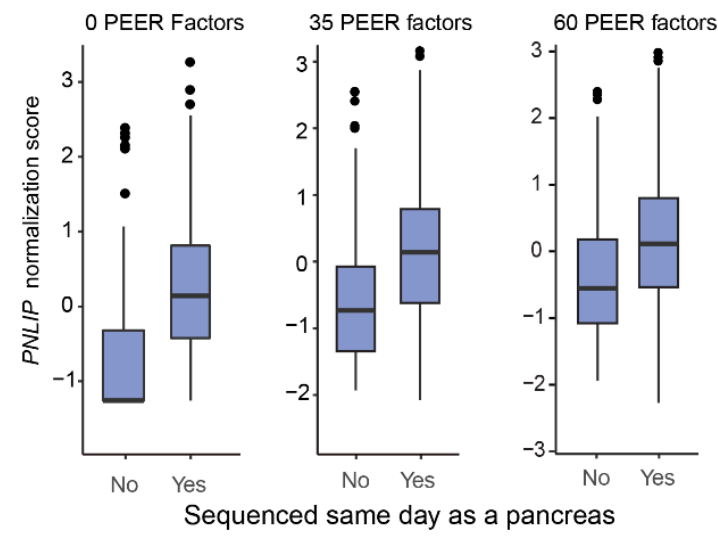

e

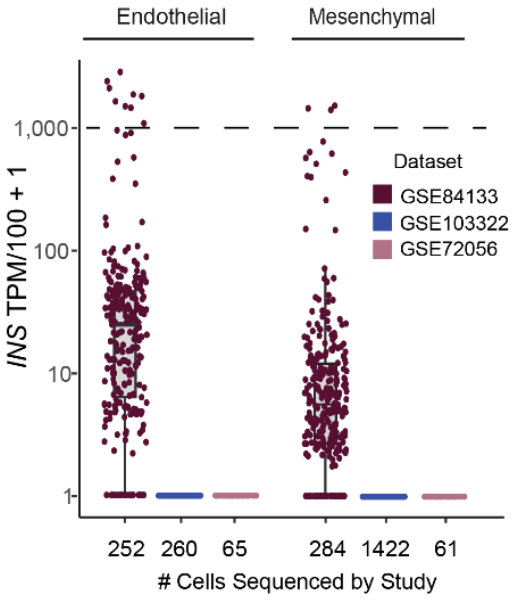

246

247

248

249

250

251

252

253

Fig. 3 Impact of PEER factors on contamination. a The top two PEER factors separated in hospital from out of hospital deaths $(\mathrm{N}=427)$. b With no PEER factor correction there is a significant increase in PNLIP expression normalized scores in lung samples if they were sequenced on the same day as a pancreas $($ No $=96$, Yes $=331$; linear regression, $p=4.34 \mathrm{e}-14)$. After $35(\mathrm{p}=1.38 \mathrm{e}-11)$ or $60(\mathrm{p}=3.03 \mathrm{e}-06)$ PEER factor corrections, the difference remained. c $P R L$ read counts in pituitary (high), placenta (medium), and uterus (low), where $P R L$ is known to be expressed across GTEx, HPA, and the RNA Atlas. The numbers in colored boxes indicate sample sizes and the color 
254 indicates respective study. d $P R L$ contamination reads across 6 tissues from 3 studies

255 that correlate with levels of likely contamination based on the other sequenced organs.

256 e INS contamination across three scRNA-Seq data sets. Only in the pancreas dataset

257 (GSE84133), where beta cells were also sequenced, does INS appear to be lowly

258 expressed in endothelial and mesenchymal cells. Cells with expression above the

259 dotted line at 1,000 TPM/100 are likely doublets or multiplets.

Contamination affects GTEx eQTL reporting

261 Using the GTEx eQTL browser, we identified 72 tissues reported as having significant

262 eQTLs for the 18 genes listed in Table 1. Seven tissues matched the known dominant

263 expression patterns of the genes. An additional 34 tissues were deemed possible based

264 on expression patterns noted by RNA and protein immunohistochemistry in which

265 expression (in TPM) was above the basal level of all tissues. However, 31 inappropriate

266 tissues were identified as harboring eQTLs even though these genes are not natively

267 expressed in these tissues, appearing only as a result of contamination (Table 4).

Table 4 Distribution of GTEx eQTLs by tissue type in contaminating genes

\begin{tabular}{|l|l|l|l|}
\hline \multicolumn{1}{|c|}{ Genes } & \multicolumn{1}{c|}{$\begin{array}{c}\text { Appropriate } \\
\text { Tissues }\end{array}$} & \multicolumn{1}{c|}{ Possible tissues } & \multicolumn{1}{c|}{ Inappropriate tissues } \\
\hline PRSS1 & -- & Small intestine & Liver, coronary, skin, lung \\
\hline PNLIP & -- & -- & -- \\
\hline CPA1 & -- & -- & Coronary \\
\hline GP2 & -- & -- & Brain \\
\hline CELA3A & Pancreas & Stomach & Liver \\
\hline KRT13 & Vagina & Lung & -- \\
\hline$P G C$ & -- & Lung, pancreas & Tibial artery \\
\hline$K R T 4$ & Esophagus & Skin, lung & Colon, brain, thyroid \\
\hline$P R L$ & -- & -- & $\begin{array}{l}\text { Gastroesophageal } \\
\text { junction, skin, tibial artery }\end{array}$ \\
\hline LIPF & & -- & -- \\
\hline$C L P S$ & Stomach & -- & -- \\
\hline$C T R B 2$ & Pancreas & -- & Aorta, brain, lung, thyroid \\
\hline
\end{tabular}




\begin{tabular}{|l|l|l|l|}
\hline FGA & Liver & Stomach & -- \\
\hline HP & -- & $\begin{array}{l}\text { Whole blood, adipose } \\
(2), \text { artery (3), lung, } \\
\text { tibial nerve, heart }\end{array}$ & Brain, esophagus mucosa \\
\hline CKM & -- & -- & Aorta, whole blood \\
\hline MYBPC1 & -- & $\begin{array}{l}\text { Heart, testis, colon, } \\
\text { prostate, brain (2) }\end{array}$ & $\begin{array}{l}\text { Esophagus (2), lung, } \\
\text { thyroid }\end{array}$ \\
\hline MYH2 & -- & -- & Colon, lung \\
\hline ZG16B & -- & $\begin{array}{l}\text { Skin (2), adrenal, } \\
\text { fibroblasts, lung, } \\
\text { stomach, prostate, } \\
\text { spleen, colon, testis, } \\
\text { whole blood }\end{array}$ & $\begin{array}{l}\text { Adipose, esophagus, } \\
\text { pituitary }\end{array}$ \\
\hline
\end{tabular}

273 multiple organs/tissues ${ }^{24,25,26}$. Neither study sequenced the pituitary, which is the organ

274 with the highest levels of prolactin (PRL) expression (Fig. 3c). Both studies performed

275 multiplexed sequencing on Illumina 2000 or 3000 sequencers. These data sets demonstrate the extent of $P R L$ expression contamination across six tissues is dependent on the amount of $P R L$ expressed in the appropriate tissues (GTEx pituitary

278 median 54,500 TPM and uterus median 4.01 TPM (Fig. 3d). To additionally characterize

279 the breadth of contamination in the literature, we identified ten studies with two or more

280 organs/cells sequenced together. All ten had cross-sample contamination of the type

281 described herein (Supplementary Data 6). We also analyzed the METSIM study,

282 containing bulk sequencing of adipose tissue in 434 subjects. ${ }^{27}$ The data from that study

283 demonstrates how, in the absence of sequencing at the same time as a contaminating

284 tissue (ex. stomach, pancreas), contaminating reads are minimal do not correlate. This

285 was opposite that observed in GTEx adipose tissue (Supplementary Fig. 3). 
We then investigated this type of highly-expressed gene contamination in

292 contamination concerned herein would not be expected to generate large numbers of

293 contaminating reads. However, investigations across two cell types, endothelial cells

294 and mesenchymal cells (fibroblasts, activated stellate cells, cancer-associated

295 fibroblasts), suggested otherwise.

296 Three different scRNA-Seq data sets (GSE84133, GSE103322, GSE72056) from

297 normal pancreas, metastatic melanoma, and head and neck squamous cell carcinoma

298 were used ${ }^{29-31}$. From the pancreas sample, insulin (INS) represented a highly-

299 expressed, cell type-enriched gene that is exclusive to beta cells (TPM value of endothelial cells and mesenchymal cells from the same data set (GSE84133), but is

302 absent in endothelial and mesenchymal cells from the other data sets in which beta

303 cells were not sequenced (Fig. 3e). Contamination of pancreatic endothelial cells is 304 further supported by a recent snATAC-seq study that demonstrated closed chromatin at 305 the INS locus in pancreatic endothelial cells ${ }^{32}$. 
Tabula Muris is a single cell transcriptome atlas of 20 mouse tissues ${ }^{33}$. We

gathered endothelial cell data from 9 tissues and generated tSNE plots of this data

based on adjustment by either 6 or 10 principal components (PCs) (Supplementary Fig.

4a, b). With 10 PCs, a subset of pancreatic endothelial cells incorrectly clustered

10. HPA staining validated the localization of these proteins to acinar cells

313 (Supplementary Fig. 4c).

\section{Discussion}

The GTEx dataset represents an ideal resource to study sequence

contamination. Its $11,000+$ samples from $700+$ individuals from a diverse set of tissues

with all library preparation and sequencing performed at one center is unique. During

our initial variation analysis of 48 tissues spanning 10,294 samples, we detected a

We found that contamination is best linked to the date of sequencing for both

326 pancreas genes and esophagus genes (linear model, $p=1.3 e-14$ and $p=6.1 e-49$

327 respectively However, both due to contamination being noted in some samples that are 328 sequenced a few days apart from a possible contaminating source and the SNP-based 329 evidence, we suspect the majority of the contamination occurred during library 
330 preparation rather than the sequencing itself. Library preparation dates were not

331 documented (personal communication, GTEx Help Desk).

While the nucleic acid isolation date was only modestly associated with library preparation was completed automatically in 96 well plates with a mix of tissues and individuals to prevent batch effects ${ }^{34}$. Fluidic carryover could have occurred here. lane specific and can even occur at the creation of the indexes when multiple indexes are purified on the same high-performance liquid chromatography column ${ }^{35}$.

346 Additionally, if steps to clean libraries of free adapters/primers are not properly

347 executed, the remaining indexes can contaminate clusters in the flow cells ${ }^{11}$. Molecular

348 recombination of indexes during sequencing can also lead to faulty read assignment as 349 multiplex clusters can become contaminated by other samples that acquire the indices 350 of the native sample (index hopping). This has been demonstrated in scRNA-Seq, and 351 seems to occur more often on a HiSeq 4000 platform ${ }^{28}$. 
GTEx's use of custom i7 (later Illumina kit-based) dual indices also reduced the amount of index hopping that can occur ${ }^{34,35}$. mechanism. Ambient RNA, from disrupted ("broken") cells may be a major source of contamination, as recently described ${ }^{36}$. If true, this would occur before the library preparation and sequencing steps, would cause only a modest expression of the gene, and would not be detectable by current doublet correcting mechanisms ${ }^{13,37}$. This method of contamination might have implications for Human Cell Atlas studies, as we demonstrated it clearly affects cross-tissue comparisons of common cell types in Tabula Muris $^{33,38}$.

Using other bulk RNA-Seq data sets with similar sequencing methods and scRNA-Seq data sets, with different preparation methods, we validated that it is predominately contamination, not low-level transcription, which results in non-zero expression values in inappropriate tissues/cells (Fig. 3d,e, Supplementary Fig. 3, generalizability of this highly-expressed transcript contamination regardless of the labs in which they take place or the methods employed.

So how big is the contamination problem? It depends on how the data is to be

373 used. Fortunately, in the GTEx data, the levels are overall low with only $2.85 \%$ of

374 samples having relatively high levels of PRSS1 (TPM $>1,00)$. Thus, for many uses of 
375 GTEx data, this level is irrelevant. However, for experiments that involve differential

376

377

378

379

380

381

382

383

384

385

386

387

388

389

390

391

392

393

394

395

396

397

expression profiling, these genes will repeatedly appear due to their variable levels of contamination. We additionally note that the GTEx standard normalization pipeline using PEER factors did not entirely eliminate this source of variation and numerous eQTLs that were identified for the 18 genes described herein were located in incorrect tissues (>40\%). We caution that our results do not suggest that one should design a study in which sample type (e.g. tissue type or disease state) is perfectly confounded with library preparation or sequencing date. In fact, it was only because the GTEx study sequenced samples from different individuals on the same date that we were able to definitively show that contamination between samples occurred.

In other scenarios, this basal contamination may be more worrisome. Many publications have reported rare, but variable gene expression in their samples claiming their importance or disease-related behaviors ${ }^{39}$. Our findings call these reports into question. The extent of cross-contamination, where one laboratories' samples are prepped and sequenced at the same time as a different laboratories' unrelated samples through a university core sequencing facility or sequencing company is unknown, but likely frequent ${ }^{40,41}$. The xenomiR story, that rice miRNAs are found in human blood through dietary means ${ }^{42}$, was shown to result from library preparation contamination $^{43,44}$. For scRNA-Seq, this contamination falsely implies some gene expression is ubiquitous across cells, which influences computational heterogeneity analyses of cell types, beyond the known challenges of other biological and technical $\operatorname{artifacts}^{33,36,45}$. Also, our work highlights the fact that work flows must be considered carefully in very-low DNA mutation detection analysis in clinical cancer samples as 
402

samples with higher tumor burdens may contaminate samples with lower tumor burdens and falsely suggest treatment approaches ${ }^{46,47}$. In particular, GTEx data is available in many outlets, including the UCSC Genome Browser. Variable, low-level expression of PRSS1, CELA3A and others may falsely intrigue researchers, particularly within the reported eQTLs.

We described low-level, variable expression contamination in the GTEx RNASeq dataset. This variation was most noticeable for 18 highly-expressed, tissueenriched genes and strongly correlates with the library preparation and sequencing of the samples. Similar contamination was observed in other bulk and scRNA-Seq data sets suggesting a universality to this type of contamination. Evaluating low-level variable gene expression in RNA sequencing data sets must be performed with precaution and awareness of potential sample contamination.

\section{Methods}

\section{Ethics Statement}

All human data was publicly available or used with approval of the GTEx consortium.

Consent was obtained by those studies.

\section{Retrieval of GTEx RNA-Seq dataset, FASTQ files, and sample data}

The gene read counts of the RNA-Seq GTEx version 7 dataset (GTEx_Analysis_201601-15_v7_RNASeQCv1.1.8_gene_reads.gct.gz) were downloaded from the GTEx Portal (https://gtexportal.org/home/datasets), along with the de-identified sample annotations (GTEx_v7_Annotations_SampleAttributesDS.txt). The FASTQ files of the 
419 tissue samples and the variant call file (VCF) files of appropriate individuals were

420 downloaded from dbGaP (phs000424.v7.p2) with the required permissions.

421 Retrieval of Human Protein Atlas tissue enriched gene list

422 We obtained the HPA tissue enriched genes by downloading a CSV file from this filtered

423 site

424 (https://www.proteinatlas.org/search/tissue specificity rna:any;Tissue\%20enriched+AN

425 D+sort by:tissue+specific+score, visited on 6/21/18).

426 Second highest tissue expression of tissue enriched genes and expression in

$427 \quad$ single tissue studies

428 Using the GTEx portal (v7) we noted the TPM for the second highest expressing tissue

429 with greater than 60 samples. We then used GEO, SRA Run Selector, and Recount2

430 (https://jhubiostatistics.shinyapps.io/recount/) to find datasets where only one tissue was

431 sequenced, with the exception of the ovary sample which was obtained from the

432 Illumina BodyMap (16 tissue types, absent of pancreas) ${ }^{48}$. Due to our inability to find

433 salivary gland data we used prostate tissue, the third highest expressing tissue for

434 ZG16B. Heart (ERP009437), adipose tissue (SRP053101), lung (SRP032833), prostate

435 (SRP003611), stomach (ERP010889), and colon (SRP029880) TPMs were acquired

436 through Recount2 and were selected for normal samples. The testis data was

437 downloaded from GEO Omnibus (GSE103905). Vagina and ovary FASTQ data was

438 downloaded from SRA run selector (GSE68229 and GSE30611). FASTQ files were

439 mapped to the Genome Reference Consortium Human Build 38 (GRCh38) using the

440 software HISAT2 version 2.1.0 ${ }^{49}$. The output SAM files were turned into BAM files and 
441 indexed using samtools version $1.9^{50,51}$. Assembly was completed using StringTie

442 v1.3.4d and StringTie TPMs were used from the output ${ }^{52,53}$.

443 Bulk sequencing processing

444

The acquired raw read counts were segmented into separate tissue subsets (48

445 tissues with $\geq 70$ samples each) and their read counts were normalized using the

446 Variance Stabilizing Transformation feature in DESeq2 version 1.22.1 in R version 3.6.1

$447{ }^{18}$. This method incorporates estimated size factors based on the median-ratio method,

448 and transformed by the dispersion-mean relationship. We then filtered the 56,202 genes

449 based on their mean expression (mean transformed count $>5$ ) to reduce noise and

450 lessen the inflated effect of low expressing genes on correlations.

451

452

453

454

455

456

457

458

459

460

461

462

\section{Identification of highly variable genes and clusters}

All analyses were completed in $\mathrm{R}$ version 3.6.1. In each tissue, a threshold of a >4 variance of variance stabilizing transformed normalized read counts was used as our cut off for highly variable transcripts. These genes were then clustered using hierarchal clustering on a distance generated by 1 - Kendall's rank-correlation coefficient. A tau critical value was calculated based on the number of samples and genes expressed. The correlation-based dendrogram was cut to produce gene clusters with average within cluster correlation of at least the tau critical value.

\section{Calculation of normalized expression scores}

Normalized expression scores allow one to summarize the expression of a gene cluster in a sample by the average normalized score of the genes in that cluster. These normalized scores were calculated by subtracting the mean expression and dividing by 
463 the median absolute deviation of the expression values for each gene across all

464 samples within a given tissue. The equation is as follows, where $x$ is the VST

465 normalized expression of gene $j$ in sample $i, t(i)$ is the tissue type for sample $i$, and $J$ is 466 the number of genes in a given cluster:

$$
S_{i}=\frac{1}{J} \sum_{j=1}^{J} \frac{x_{i j}-\bar{x}_{j}}{\operatorname{Median}\left|x_{m j}-\bar{x}_{j}\right|}
$$

A "pancreas" contamination score was defined as the average normalized score using the genes PRSS1, CELA3A, PNLIP, and CLPS, across all tissues. An

"esophagus" normalized score was defined as the average normalized score using genes KRT4 and KRT13 in a given tissue. All normalized scores were calculated

471 independently for each tissue, to account for tissue-specific between-sample variation in

472 the expression of these genes.

\section{Linear mixed model analysis for pancreas gene contamination}

475 sequencing on the same day as a potential contaminating tissue (source of gene

477 in package Ime4 (v1.1-21). In the linear mixed models we used all available tissues with

$478>40$ samples not sequenced on the same day as a pancreas sample (15 tissues,

$479 \mathrm{~N}=6,258)$. For the model, tissue was used as a covariate along with "pancreas

480 sequencing day" and "pancreas nucleic acid isolation day" were coded binarily as

481 True/False based on whether a given non-pancreas sample was sequenced or 
482

483

484

485

486

487

488

489

490

491

492

493

494

495

496

497

underwent RNA isolation on the same day as a pancreas sample. Our model identified an association between the pancreas contamination score with pancreas sequencing day $(T / F ; 1 / 0)$, pancreas nucleic acid isolation day $(T / F ; 1 / 0)$, and tissue as covariates, with the subject IDs included as a random effect. The equation for our model is below where $t(i)$ gives the tissue type of sample $i, s(i)$ is defined as the sequencing day of the sample, and $d(i)$ is the isolation date of sample $i$. Lastly $T$ is the number of tissue types, $u_{n(i)}$ is the random effect of subject ID for sample $i$, and $\varepsilon_{i}$ is the error term for said sample:

$$
\begin{aligned}
& Y_{i}=\beta_{0}+\beta_{1} I\left(s(i) \in\left\{s\left(m: t(m)=t_{\text {pancreas }}\right)\right\}\right) \\
& +\beta_{2} I\left(d(i) \in\left\{d\left(m: t(m)=t_{\text {pancreas }}\right)\right\}\right) \\
& +\sum_{k=3}^{T+1} \beta_{k} I(t(i)=k-1)+u_{n(i)}+\varepsilon_{i}
\end{aligned}
$$

\section{Linear mixed model analysis for esophagus gene contamination}

We repeated the above analysis with the esophagus mucosa, coding shared sequencing day and shared nucleic acid isolation day similarly. In the linear mixed model we used all tissues with a sample size $>40$ samples not sequenced on the same day as the esophagus mucosa ( 8 tissues, $\mathrm{N}=3,917)$. The model used esophagus nucleic acid isolation day (T/F; 1/0), esophagus sequencing day (T/F; 1/0), and tissue as covariates along with subject IDs as a random effect on the esophagus contamination score.

\section{Base pair incongruency analysis}


Base pair incongruency analysis required a contaminated tissue expression

500

501

502

503

504

505

506

507

508

509

510

511

512

513

514

515

516

517

518

519

520

521 FASTQ, a native tissue expression FASTQ, and the individual's VCF file. FASTQ files were mapped to the Genome Reference Consortium Human Build 37 (hg19) using the software HISAT2 version 2.1.0 ${ }^{49}$. The output SAM files were turned into BAM files and indexed using samtools version $1.9^{50,51}$. Preliminary analysis and development of figures were generated using the Integrative Genome Viewer version 2.4.13 ${ }^{54,55}$ ). Protein coding SNPs, rare variants, and personal variants (collectively referred to as variants in this paper), were manually selected using IGV as a reference. Using the tool bam-readcount version 0.8.0 in combination with a Python 3.6.2 script, a list of RNASeq and genomic incongruencies were generated for the acquired sample BAM files.

\section{PEER factor analysis}

We obtained the GTEx RNA-Seq dataset from lung $(\mathrm{N}=427)$. The data underwent trimmed mean of m-values (TMM) normalization and filtering out of lowly expressed genes ( $<0.1$ TPM for $80 \%$ or more of the samples) before running PEER to identify potential confounders ${ }^{22}$. Following GTEx's pipeline

\section{(https://gtexportal.org/home/documentationPage\#staticTextAnalysisMethods visited),}

we then performed an inverse normal transformation (INT) on the expression values for each gene in order to reduce the effect of outliers ${ }^{23}$. Normalized scores for each gene are based on TMM-normalization, inverse-normal transformation, and scaling/centering at zero. A linear regression was performed, with either raw inverse-normalized expression values or expression normalized scores (after correcting for PEER factors) as the outcomes and same-day pancreas (/esophagus) sequencing status as the predictor. Beta estimates represent how many standard deviations greater the mean 
522 expression of a gene is when samples are sequenced on the same day as a

523 contaminating tissue, even when accounting for variance explained by 60 PEER factors.

\section{Cross-referencing eQTLs with contamination findings}

We obtained and tallied eQTL reports for the 18 genes in Table 1 from the GTEx

526 eQTL browser (https://gtexportal.org visited on March 26, 2019). eQTLs were identified

527 by tissue association and conservatively placed in one of three categories: appropriate

528 expression, possible expression, and inappropriate expression. The appropriateness of

529 expression in any tissue was based on the evaluation of TPM levels in the tissue and

530 immunohistochemistry staining patterns as noted in the HPA ${ }^{56}$.

531 Acquiring Human Protein Atlas, RNA Atlas RNA-Seq and METSIM Data

533 accession ERP003613 ${ }^{24}$. The RNA Atlas was acquired by downloading their raw RNA-

534 seq counts from Gene Expression Omnibus (GSE120795) ${ }^{25}$. The HPA RNA-Seq was

535 performed across 27 tissues and the RNA Atlas was across 20 tissues. We filtered

536 samples down to the shared tissues of liver, lung, pancreas, prostate, skin, and

537 stomach as well as the potential sources of $P R L$ contamination of pituitary, placenta and

538 uterus. Only sun exposed skin was used for GTEx analysis. TPM RNA-seq data from

539 the METSIM study was downloaded through the GEO Omnibus (GSE135134) ${ }^{27}$. 
We identified three publications with human scRNA-Seq data sets that all

543 contained endothelial and mesenchymal cells ${ }^{29-31}$. All three sets had median read

544 counts/cell $>100,000$. The processed read counts from the three studies were obtained

545 from the Gene Expression Omnibus (GEO) (GSE84133; GSE103322; GSE72056). For

546 each study we used the supplied cell type information to identify endothelial and

547 mesenchymal-type cells. Mesenchymal cells were labeled as "activated stellate"

548 (GSE84133), "fibroblast” (GSE103322), and “cancer-associated fibroblast” (GSE72056).

549 The GSE103322 and GSE72056 read data were generated using Smart-Seq2 on a

550 NextSeq 500 instrument $^{57}$. Raw FASTQ data sets were normalized using RSEM to

551 calculate $E_{i, j}=\log _{2}\left(T P M_{i, j} / 10+1\right)$, where $T P M_{i, j}$ refers to transcript-per-million for gene $\mathrm{i}$ in

552 sample $\mathrm{j}^{58}$. The $\mathrm{TPM}_{\mathrm{i}, \mathrm{j}}$ was then divided by 10 , to be on the order of 100,000 transcripts

553 to account for single cell read depth and the effect of 0 s in downstream analysis ${ }^{29}$.

554 GSE84133 was generated using the inDrop method and sequenced on a HiSeq 2500

555 instrument ${ }^{59}$. Human islets were isolated using a modified Ricordi method which

556 includes collagenase, mechanical agitation, pumping and centrifugation ${ }^{60}$. To make

557 single cells, the islets were then centrifuged twice at $250 \mathrm{rpm}$ and treated with TrypLE

558 Express before mechanical dispersion with a P100 pipette, followed by centrifugation at

$559500 \mathrm{rpm}$. The two cancer sample libraries (GSE103322, GSE72056) were generated

560 using Smart-Seq2 and sequenced on a NextSeq 500 instrument ${ }^{57}$. Read counts were

561 scaled using TPM/100 to equalize expression level counts to the other two studies. The

562 calculation of the percent contamination of endothelial cells by INS was the ratio of

563 average normalized INS read counts per endothelial cell divided by the normalized

564 average read count in beta cells and excluding the 9 cells with >1000 TPM/100. 
We obtained the Tabula Muris read data from

566 https://figshare.com/articles/Robject_files_for_tissues_processed_by_Seurat/5821263

567 (visited on 8/8/19) ${ }^{33}$. We used the R package Seurat (v3.1.1) and selected endothelial

568 cells from nine organs/tissues (brain, fat, heart, kidney, limb muscle, liver, lung,

569 pancreas, trachea) based on the annotation of the seurat object in the metadata slot

570 cell_ontology_class labeled 'endothelial cell'61. tSNE plots were generated based on the

571 top 6 or top 10 PCs. The average number of PCs used by Tabula Muris per

572 tissue/organ to create tSNE maps was 12 and the median was 10. Images of protein

573 expression of contaminating pancreas genes were obtained from the HPA ${ }^{56}$.

576 Data availability

577 No new sequencing data was created for this study. All data used in this study is

578 available through dbGap (https://www.ncbi.nlm.nih.gov/gap/), GEO

579 (https://www.ncbi.nlm.nih.gov/gds), recount2

580 (https://lcolladotor.github.io/project/recount2/) or Tabula Muris

581 (https://figshare.com/articles/Robject files for tissues processed by Seurat/5821263). 
584 Code for all analyses is deposited at GitHub

585 (https://github.com/mhalushka/gtex contamination code). Computational analyses

586 were done using public R packages except when specifically noted otherwise.

\section{Acknowledgements}

589 The authors thank the GTEx Help Desk, Kristen Ardlie, Sheila Dodge, and NDRI for

590 valuable assistance in understanding the technical data sets and islet isolation

591 methodology. M.K.H. was supported by grants 1R01HL137811, R01GM130564, and

592 P30CA006973 from the National Institutes of Health and 17GRNT33670405 from the

593 American Heart Association. T.O.N. was supported by grants R01GM130564 and

594 T32GM07814. D.E.A was supported by 1R01HL131573 and 1R01HL137811. M.N.M.

595 was supported by R01HL137811 and the University of Rochester CTSA award number

596 UL1TR002001. A.Z.R was supported by R01GM130564.

597 Contributions: M.K.H., M.N.M and A.Z.R conceived of the experiments and assisted

598 with the manuscripts. T.O.N. performed the experiments, analyzed the data and wrote

599 the manuscript. S.Y., R.X.V., V.P. and D.E.A. performed experiments and assisted on

600 the manuscript.

601 Conflicts of interest

602 The authors declare no conflicts of interest.

603

604

\section{References}


605

606

607

608

609

610

611

612

613

614

615

616

617

618

619

620

621

622

623

624

625

626

627

628

629

630

631

632

633

634

635

636

637

638

639

640

641

642

643

644

645

646

647

648

649

650

651

1 Consortium, G. T. The Genotype-Tissue Expression (GTEx) project. Nat Genet 45, 580-585, doi:10.1038/ng.2653 (2013).

2 Tomczak, K., Czerwinska, P. \& Wiznerowicz, M. The Cancer Genome Atlas (TCGA): an immeasurable source of knowledge. Contemporary oncology 19, A68-77, doi:10.5114/wo.2014.47136 (2015).

3 Zeisel, A. et al. Brain structure. Cell types in the mouse cortex and hippocampus revealed by single-cell RNA-seq. Science 347, 1138-1142, doi:10.1126/science.aaa1934 (2015).

4 Kumasaka, N., Knights, A. J. \& Gaffney, D. J. Fine-mapping cellular QTLs with RASQUAL and ATAC-seq. Nat Genet 48, 206-213, doi:10.1038/ng.3467 (2016).

5 Gutman, D. A. et al. MR imaging predictors of molecular profile and survival: multi-institutional study of the TCGA glioblastoma data set. Radiology 267, 560-569, doi:10.1148/radiol.13120118 (2013).

6 Ewing, A. D. et al. Combining tumor genome simulation with crowdsourcing to benchmark somatic single-nucleotide-variant detection. Nature methods 12, 623-630, doi:10.1038/nmeth.3407 (2015).

7 Okoniewski, M. J. \& Miller, C. J. Hybridization interactions between probesets in short oligo microarrays lead to spurious correlations. BMC Bioinformatics 7, 276, doi:10.1186/1471-2105-7276 (2006).

8 van Dijk, E. L., Jaszczyszyn, Y. \& Thermes, C. Library preparation methods for next-generation sequencing: tone down the bias. Experimental cell research 322, 12-20, doi:10.1016/j.yexcr.2014.01.008 (2014).

9 Tuerk, A., Wiktorin, G. \& Guler, S. Mixture models reveal multiple positional bias types in RNASeq data and lead to accurate transcript concentration estimates. PLoS computational biology 13, e1005515, doi:10.1371/journal.pcbi.1005515 (2017).

10 Lusk, R. W. Diverse and widespread contamination evident in the unmapped depths of high throughput sequencing data. PLoS One 9, e110808, doi:10.1371/journal.pone.0110808 (2014).

11 Illumina. Effects of Index Misassignment on Multiplexing and Downstream Analysis, $<$ https://www.illumina.com/content/dam/illuminamarketing/documents/products/whitepapers/index-hopping-white-paper-770-2017-004.pdf> (

12 Rosenberg, A. Z. et al. xMD-miRNA-seq to generate near in vivo miRNA expression estimates in colon epithelial cells. Scientific reports 8, 9783, doi:10.1038/s41598-018-28198-z (2018).

13 McGinnis, C. S., Murrow, L. M. \& Gartner, Z. J. DoubletFinder: Doublet Detection in Single-Cell RNA Sequencing Data Using Artificial Nearest Neighbors. Cell Syst 8, 329-337 e324, doi:10.1016/j.cels.2019.03.003 (2019).

14 Merchant, S., Wood, D. E. \& Salzberg, S. L. Unexpected cross-species contamination in genome sequencing projects. PeerJ 2, e675, doi:10.7717/peerj.675 (2014).

15 Cibulskis, K. et al. ContEst: estimating cross-contamination of human samples in next-generation sequencing data. Bioinformatics 27, 2601-2602, doi:10.1093/bioinformatics/btr446 (2011).

$16 \mathrm{Ma}, \mathrm{X}$. et al. Analysis of error profiles in deep next-generation sequencing data. Genome Biol 20 , 50, doi:10.1186/s13059-019-1659-6 (2019).

17 McCall, M. N., Illei, P. B. \& Halushka, M. K. Complex Sources of Variation in Tissue Expression Data: Analysis of the GTEx Lung Transcriptome. American journal of human genetics 99, 624635, doi:10.1016/j.ajhg.2016.07.007 (2016).

18 Love, M. I., Huber, W. \& Anders, S. Moderated estimation of fold change and dispersion for RNA-seq data with DESeq2. Genome Biol 15, 550, doi:10.1186/s13059-014-0550-8 (2014).

19 Muraro, M. J. et al. A Single-Cell Transcriptome Atlas of the Human Pancreas. Cell Syst 3, 385394 e383, doi:10.1016/j.cels.2016.09.002 (2016). 
Witwer, K. W. \& Halushka, M. K. Toward the promise of microRNAs - Enhancing reproducibility and rigor in microRNA research. RNA biology 13, 1103-1116, doi:10.1080/15476286.2016.1236172 (2016).

40 Kryukov, K. \& Imanishi, T. Human Contamination in Public Genome Assemblies. PLoS One 11, e0162424, doi:10.1371/journal.pone.0162424 (2016).

41 Longo, M. S., O'Neill, M. J. \& O'Neill, R. J. Abundant human DNA contamination identified in nonprimate genome databases. PLoS One 6, e16410, doi:10.1371/journal.pone.0016410 (2011).

42 Zhang, L. et al. Exogenous plant MIR168a specifically targets mammalian LDLRAP1: evidence of cross-kingdom regulation by microRNA. Cell Res 22, 107-126, doi:10.1038/cr.2011.158 (2012).

43 Tosar, J. P., Rovira, C., Naya, H. \& Cayota, A. Mining of public sequencing databases supports a non-dietary origin for putative foreign miRNAs: underestimated effects of contamination in NGS. RNA 20, 754-757, doi:10.1261/rna.044263.114 (2014).

44 Zhang, Y. et al. Analysis of plant-derived miRNAs in animal small RNA datasets. BMC Genomics 13, 381, doi:10.1186/1471-2164-13-381 (2012).

$45 \mathrm{Li}, \mathrm{H}$. et al. Reference component analysis of single-cell transcriptomes elucidates cellular heterogeneity in human colorectal tumors. Nat Genet 49, 708-718, doi:10.1038/ng.3818 (2017).

46 Tian, S. K. et al. Optimizing Workflows and Processing of Cytologic Samples for Comprehensive Analysis by Next-Generation Sequencing: Memorial Sloan Kettering Cancer Center Experience. Archives of pathology \& laboratory medicine, doi:10.5858/arpa.2016-0108-RA (2016).

47 Van Allen, E. M. et al. Whole-exome sequencing and clinical interpretation of formalin-fixed, paraffin-embedded tumor samples to guide precision cancer medicine. Nature medicine 20, 682-688, doi:10.1038/nm.3559 (2014).

48 Collado-Torres, L., Nellore, A. \& Jaffe, A. E. recount workflow: Accessing over 70,000 human RNA-seq samples with Bioconductor. F1000Research 6, 1558, doi:10.12688/f1000research.12223.1 (2017).

49 Kim, D., Langmead, B. \& Salzberg, S. L. HISAT: a fast spliced aligner with low memory requirements. Nature methods 12, 357-360, doi:10.1038/nmeth.3317 (2015).

$50 \mathrm{Li}, \mathrm{H}$. et al. The Sequence Alignment/Map format and SAM tools. Bioinformatics 25, 2078-2079, doi:10.1093/bioinformatics/btp352 (2009).

$51 \mathrm{Li}, \mathrm{H}$. A statistical framework for SNP calling, mutation discovery, association mapping and population genetical parameter estimation from sequencing data. Bioinformatics 27, 2987-2993, doi:10.1093/bioinformatics/btr509 (2011).

52 Pertea, M. et al. StringTie enables improved reconstruction of a transcriptome from RNA-seq reads. Nature biotechnology 33, 290-295, doi:10.1038/nbt.3122 (2015).

53 Pertea, M., Kim, D., Pertea, G. M., Leek, J. T. \& Salzberg, S. L. Transcript-level expression analysis of RNA-seq experiments with HISAT, StringTie and Ballgown. Nature protocols 11, 1650-1667, doi:10.1038/nprot.2016.095 (2016).

54 Thorvaldsdottir, H., Robinson, J. T. \& Mesirov, J. P. Integrative Genomics Viewer (IGV): highperformance genomics data visualization and exploration. Briefings in bioinformatics 14, 178 192, doi:10.1093/bib/bbs017 (2013).

55 Robinson, J. T. et al. Integrative genomics viewer. Nature biotechnology 29, 24-26, doi:10.1038/nbt.1754 (2011).

56 Uhlen, M. et al. Towards a knowledge-based Human Protein Atlas. Nature biotechnology 28, 1248-1250, doi:10.1038/nbt1210-1248 (2010).

57 Picelli, S. et al. Smart-seq2 for sensitive full-length transcriptome profiling in single cells. Nature methods 10, 1096-1098, doi:10.1038/nmeth.2639 (2013). 
bioRxiv preprint doi: https://doi.org/10.1101/602367; this version posted January 2, 2020. The copyright holder for this preprint (which was not certified by peer review) is the author/funder, who has granted bioRxiv a license to display the preprint in perpetuity. It is made available under aCC-BY-NC-ND 4.0 International license.

74458 Li, B. \& Dewey, C. N. RSEM: accurate transcript quantification from RNA-Seq data with or 745 without a reference genome. BMC Bioinformatics 12, 323, doi:10.1186/1471-2105-12-323

746 (2011).

$74759 \quad$ Klein, A. M. et al. Droplet barcoding for single-cell transcriptomics applied to embryonic stem $748 \quad$ cells. Cell 161, 1187-1201, doi:10.1016/j.cell.2015.04.044 (2015).

74960 Ricordi, C., Lacy, P. E., Finke, E. H., Olack, B. J. \& Scharp, D. W. Automated method for isolation $750 \quad$ of human pancreatic islets. Diabetes 37, 413-420 (1988).

75161 Satija, R., Farrell, J. A., Gennert, D., Schier, A. F. \& Regev, A. Spatial reconstruction of single-cell 752 gene expression data. Nature biotechnology 33, 495-502, doi:10.1038/nbt.3192 (2015). 


\title{
Basal Contamination of Sequencing: Lessons from the GTEx dataset
}

\author{
Tim O. Nieuwenhuis et al.
}

Supplementary Information 


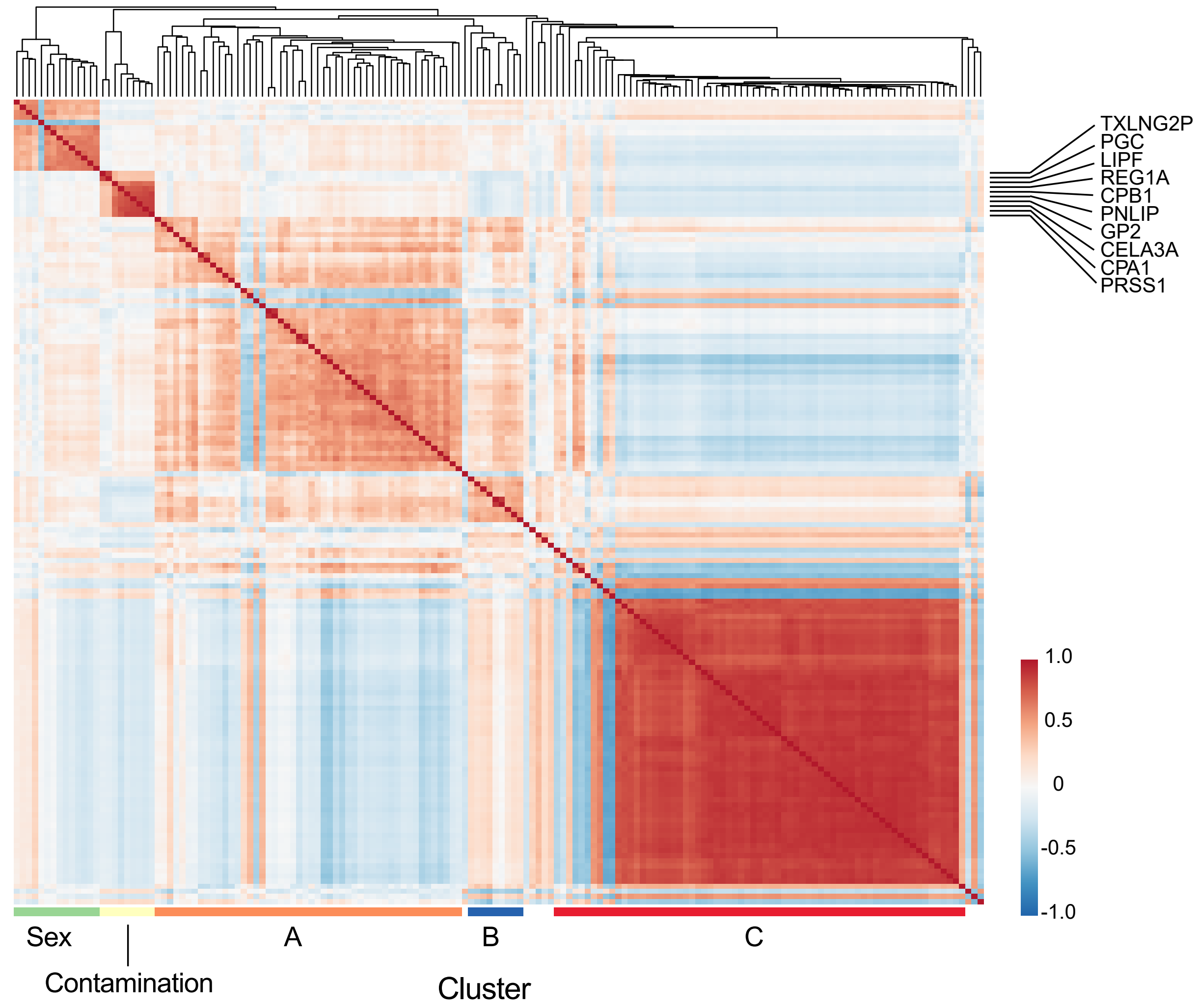


Supplementary Figure 1. A correlation heatmap of the highly variable gene clusters in 343 transformed fibroblast samples. Red shows a positive correlation. Genes within the contamination cluster are given. The etiology of clusters $A$ and $B$ are unknown. Cluster $\mathrm{C}$ represents co-variable genes related to mitosis. 


\section{Normalization score of pancreas contamination}

Pancreas Gene Cluster Identified in These Tissues

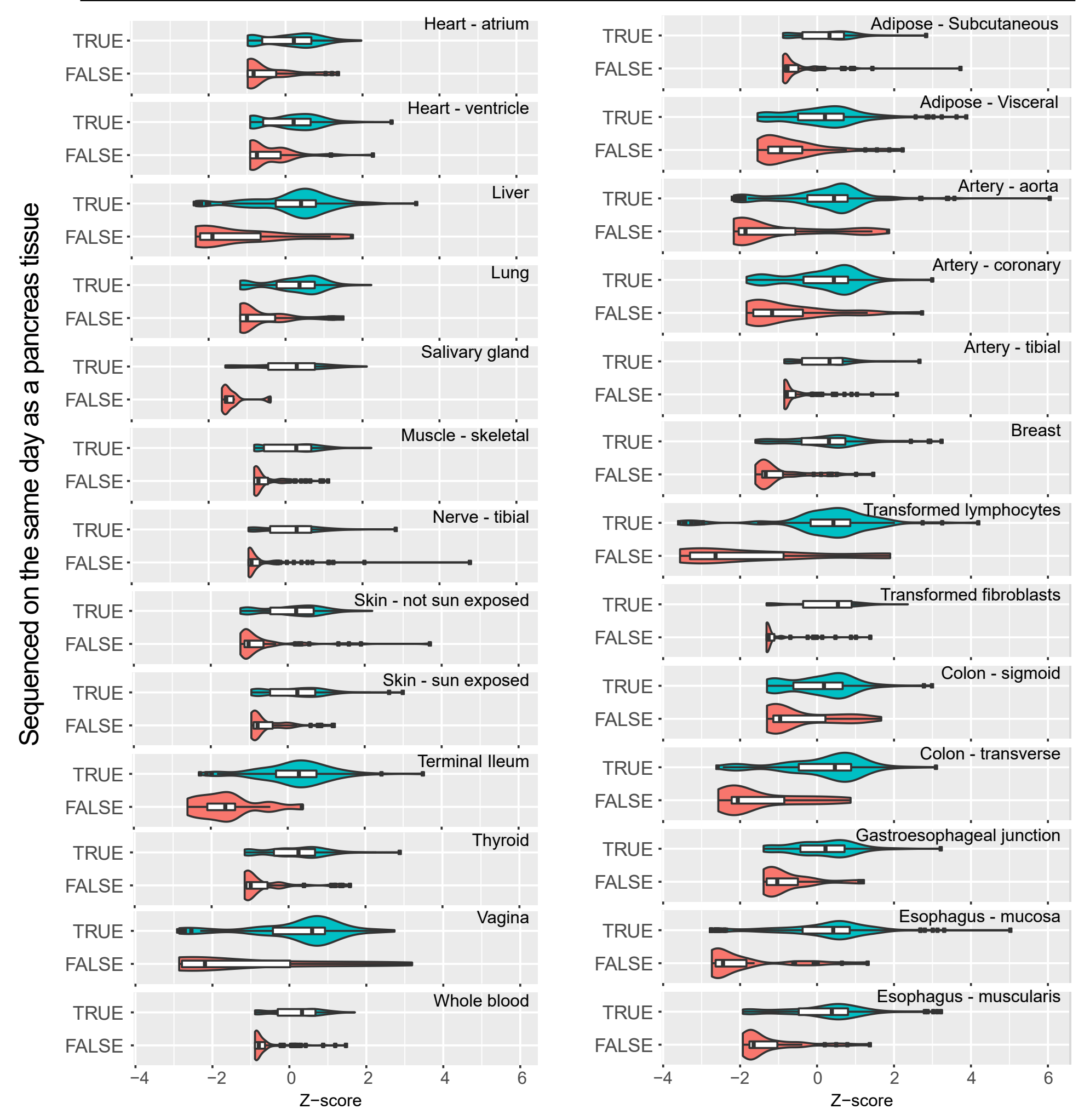

Pancreas Gene Cluster Not Identified in These Tissues

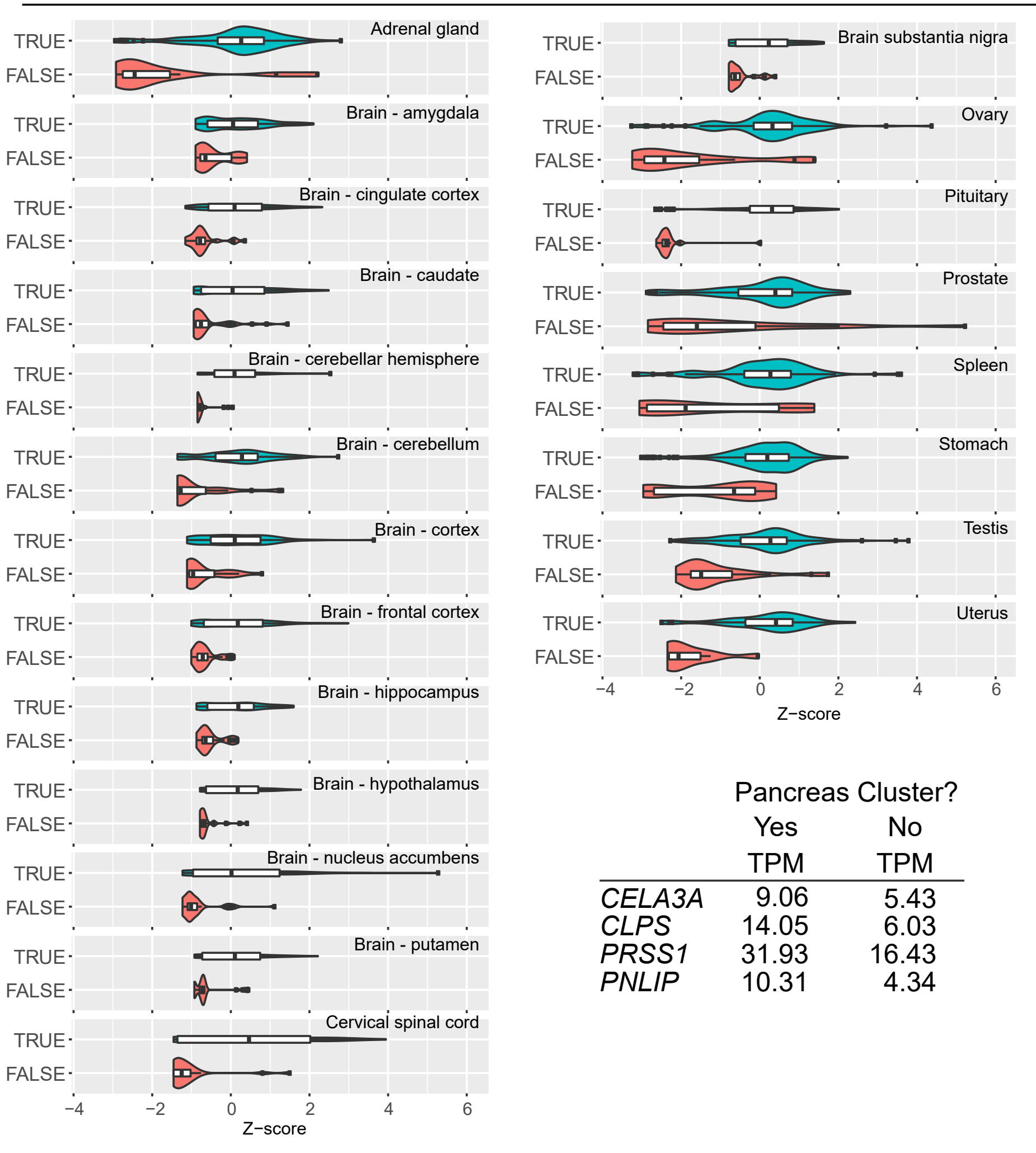


Supplementary Figure 2. Violin plots representing the pancreatic gene (CELA3A, CLPS, PRSS1, PNLIP) normalization scores of samples sequenced the same day as a pancreas sample and those that were not. The bottom right corner shows the median TPM of pancreas genes in non-pancreas tissues. These were divided base on the tissue having all four genes in their contamination cluster $(\mathrm{N}=26)$ or not $(\mathrm{N}=21)$. 
GTEx - Adipose

Pancreas genes

4. $R^{\wedge} 20.80$

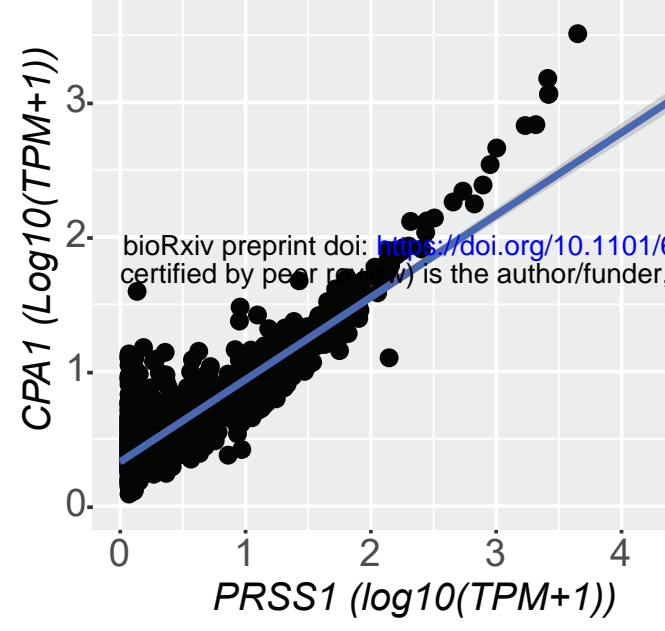

Esophagus genes

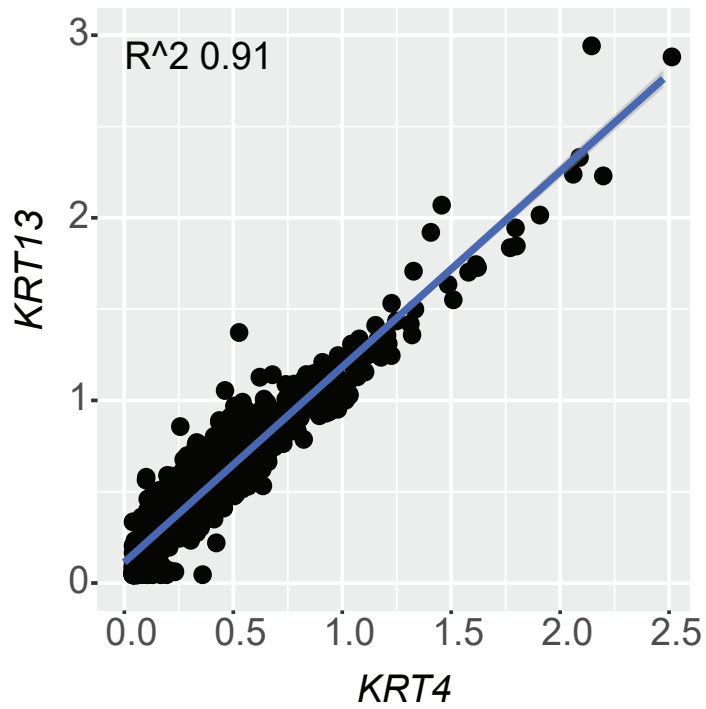

Stomach genes

3- $R^{\wedge} 20.89$

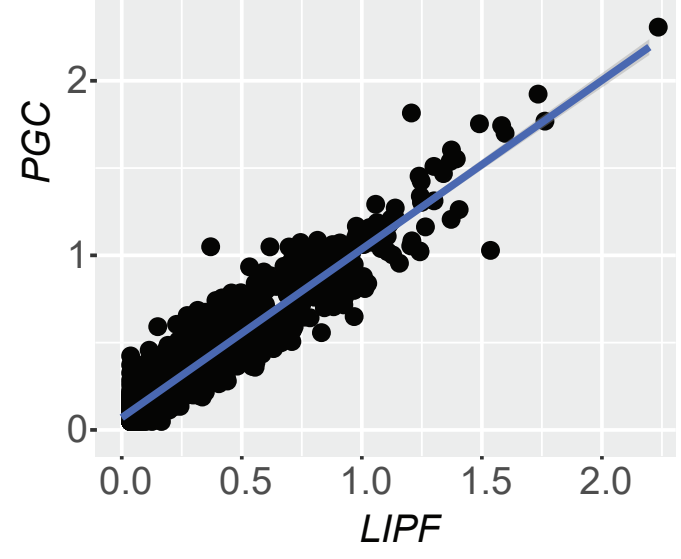

Skeletal Muscle genes

3. $\mathrm{R}^{\wedge} 20.77$

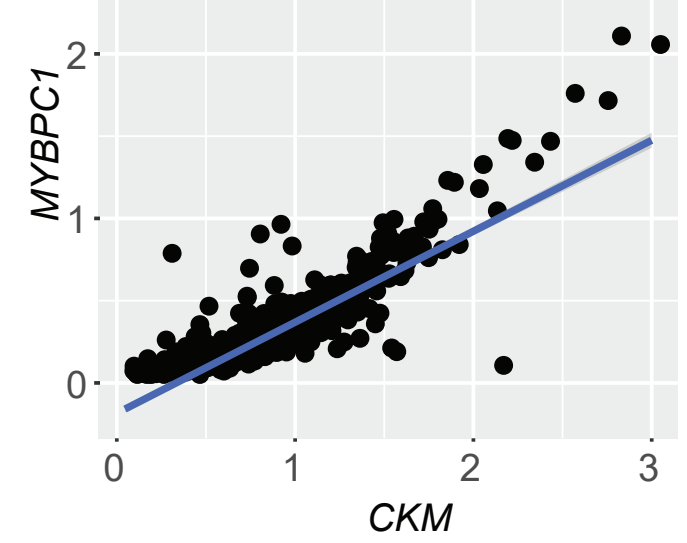

METSIM - Adipose
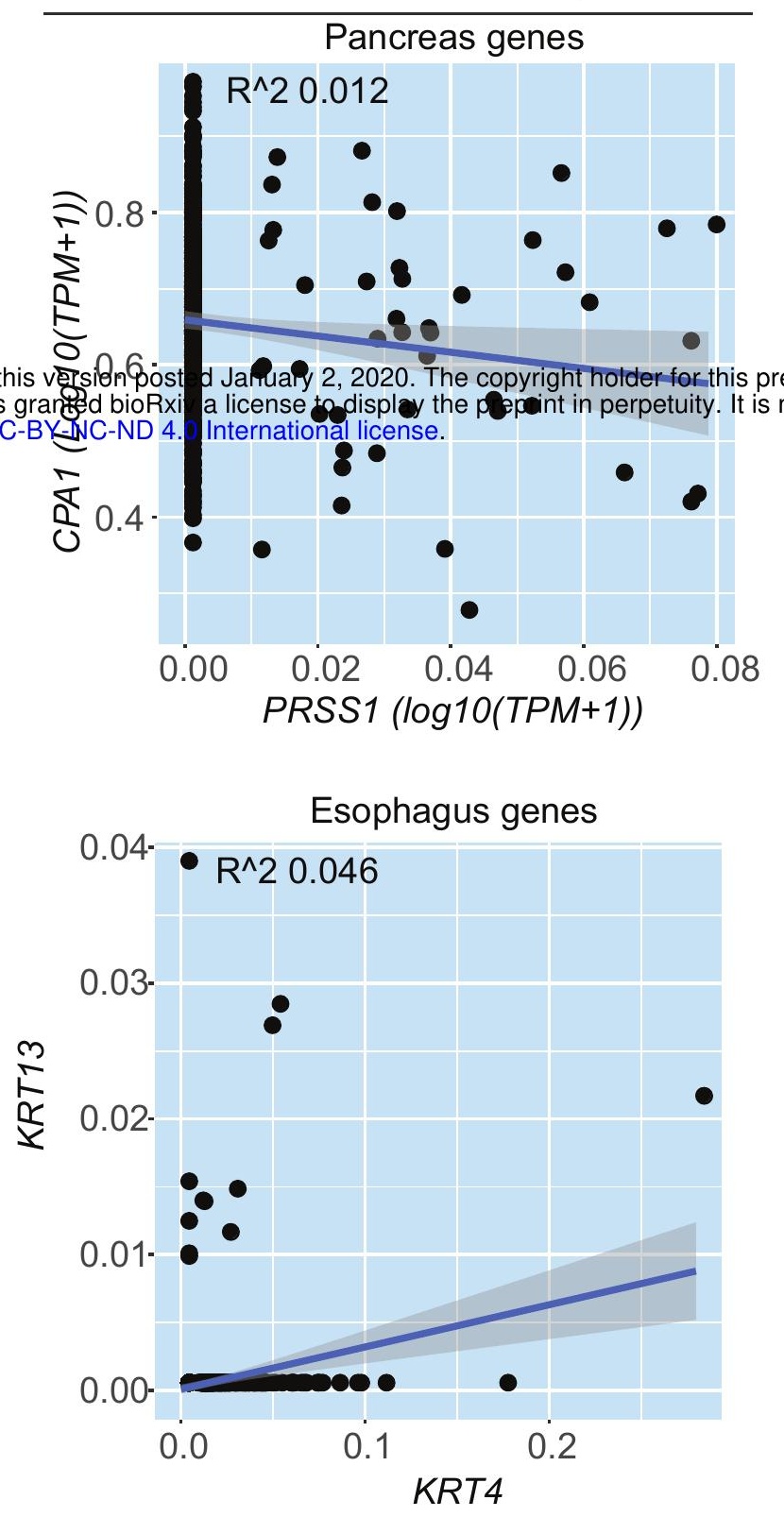

Stomach genes

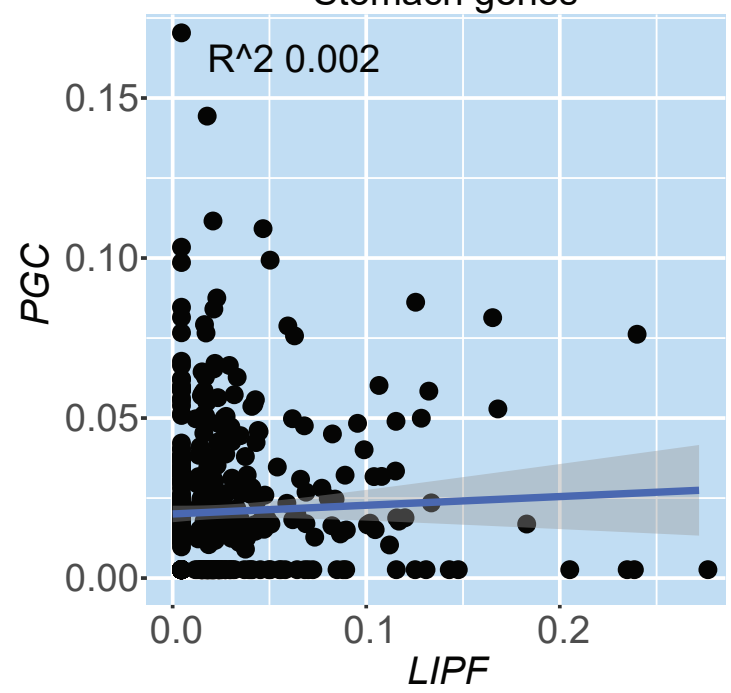

Skeletal Muscle genes

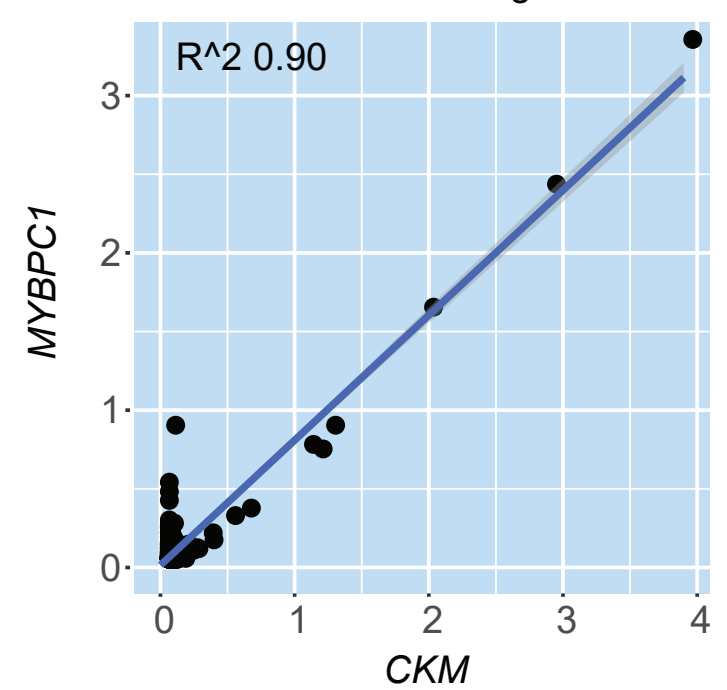


Supplementary Figure 3. A scatterplot comparison of different contaminating genes between the GTEx adipose tissues $(n=797)$ and the adipose tissues harvested in the METSIM study ( $\mathrm{n}=434)$. We compared CPA1 vs PRSS1, KRT13 vs KRT4, PGC vs $L I P F$, and MYBPC1 vs $C K M$ as each of those gene pairs are highly expressed together in a contaminating tissue type. In GTEx the expression is consistently higher and has a stronger positive correlation compared to the METSIM study for three of those pairs Because skeletal muscle can be captured in adipose harvesting, MYBPC1 and CKM also showed strong correlation in METSIM as a result of a direct contamination. 


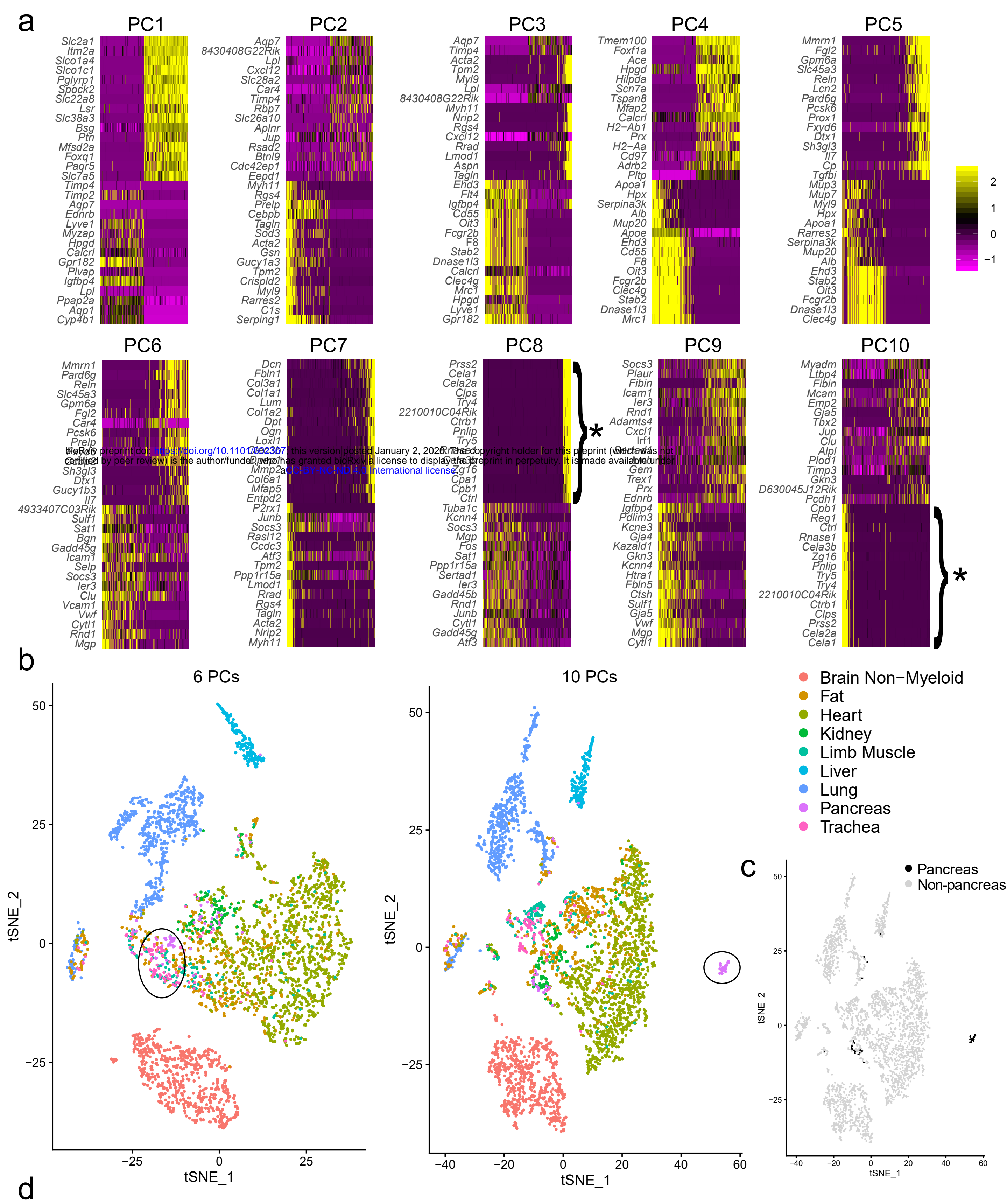

b

. - A R CELA3B

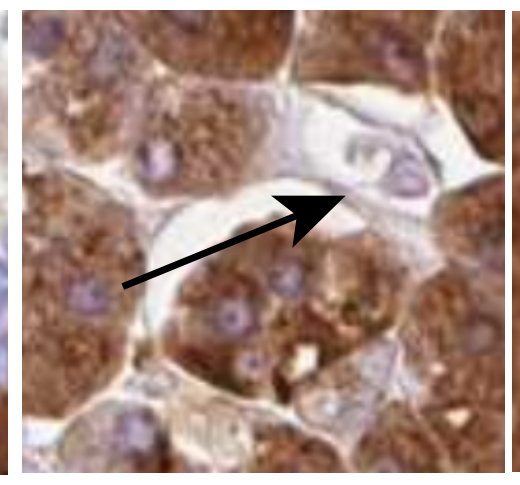
TRY4 PNLIP

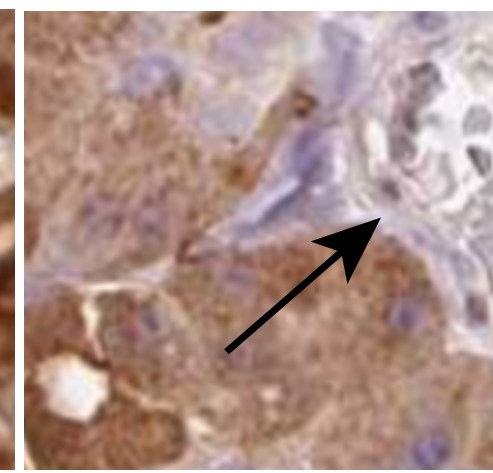

CLPS

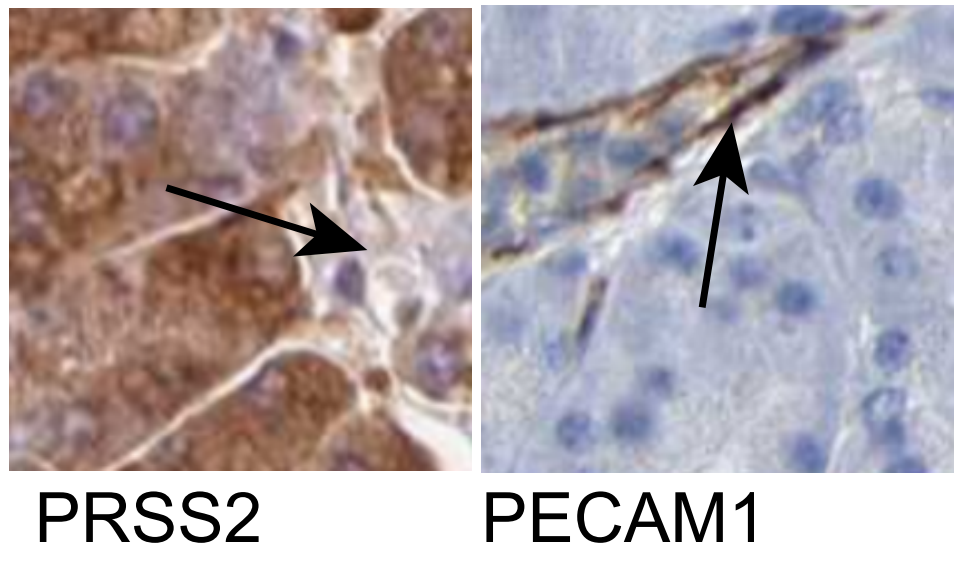


Supplementary Figure 4. Pancreatic acinar cell contamination drives endothelial cell diversity in Tabula Muris. a The major genes of the top 10 principal components (PCs) for nine groups of endothelial cells. * represents contaminating acinar cell expression. b tSNE plots based on 6 or 10 PCs demonstrate a large deviation of pancreas endothelial cells from a shared cluster to a unique cluster based on additional PCs. c In this black and white representation, a subset of pancreas cells are noted to move. $\mathbf{d}$ Representative images (HPA) of several highly-expressed acinar proteins that do not mark endothelial cells (black arrows) and PECAM1, a known endothelial cell marker. 